\title{
VLBI for Gravity Probe B. VII. The Evolution of the Radio Structure of IM Pegasi
}

\section{Citation}

Bietenholz, M. F., N. Bartel, D. E. Lebach, R. R. Ransom, M. I. Ratner, and I. I. Shapiro. 2012. VLBI for Gravity Probe B. VII. The evolution of the radio structure of IM Pegasi. The Astrophysical Journal Supplement Series 201 (1): 7.

\section{Published Version}

doi:10.1088/0067-0049/201/1/7

\section{Permanent link}

http://nrs.harvard.edu/urn-3:HUL.InstRepos:11856178

\section{Terms of Use}

This article was downloaded from Harvard University's DASH repository, and is made available under the terms and conditions applicable to Other Posted Material, as set forth at http:// nrs.harvard.edu/urn-3:HUL.InstRepos:dash.current.terms-of-use\#LAA

\section{Share Your Story}

The Harvard community has made this article openly available.

Please share how this access benefits you. Submit a story.

\section{Accessibility}




\title{
VLBI FOR GRAVITY PROBE B. VII. THE EVOLUTION OF THE RADIO STRUCTURE OF IM PEGASI
}

\author{
M. F. Bietenholz ${ }^{1,3}$, N. Bartel ${ }^{1}$, D. E. Lebach ${ }^{2}$, R. R. RANSOM ${ }^{1,4}$, M. I. RATNeR ${ }^{2}$, AND I. I. Shapiro ${ }^{2}$ \\ ${ }^{1}$ Department of Physics and Astronomy, York University, 4700 Keele Street, Toronto, ON M3J 1P3, Canada \\ ${ }^{2}$ Harvard-Smithsonian Center for Astrophysics, 60 Garden Street, Cambridge, MA 02138, USA \\ Received 2011 May 31; accepted 2011 December 20; published 2012 June 7
}

\begin{abstract}
We present measurements of the total radio flux density as well as very long baseline interferometry images of the star, IM Pegasi, which was used as the guide star for the NASA/Stanford relativity mission Gravity Probe B. We obtained flux densities and images from 35 sessions of observations at $8.4 \mathrm{GHz}(\lambda=3.6 \mathrm{~cm})$ between 1997 January and 2005 July. The observations were accurately phase-referenced to several extragalactic reference sources, and we present the images in a star-centered frame, aligned by the position of the star as derived from our fits to its orbital motion, parallax, and proper motion. Both the flux density and the morphology of IM Peg are variable. For most sessions, the emission region has a single-peaked structure, but $25 \%$ of the time, we observed a two-peaked (and on one occasion perhaps a three-peaked) structure. On average, the emission region is elongated by $1.4 \pm 0.4$ mas (FWHM), with the average direction of elongation being close to that of the sky projection of the orbit normal. The average length of the emission region is approximately equal to the diameter of the primary star. No significant correlation with the orbital phase is found for either the flux density or the direction of elongation, and no preference for any particular longitude on the star is shown by the emission region.
\end{abstract}

Key words: binaries: close - radio continuum: stars - stars: activity - stars: imaging - stars: individual (IM Pegasi) - techniques: interferometric

Online-only material: animation

\section{INTRODUCTION}

IM Pegasi (hereafter IM Peg; HR 8703, HD 216489, FK5 3829) is a close binary RS Canum Venaticorum (RS CVn) star (Hall 1976). This star was chosen as the reference star for Gravity Probe B $(G P-B)$, the space-borne relativity experiment developed by NASA and Stanford University; for an introduction to $G P-B$, see Shapiro et al. (2012), hereafter Paper I. The $G P-B$ experiment required a very accurate measurement of the proper motion of the guide star, which was determined by using phase-referenced very long baseline interferometry (VLBI) measurements relative to the compact extragalactic objects 3C 454.3, QSO B2250+194, and B2252+172. This paper is the seventh and last in a series describing the $G P-B$ experiment and the astrometric observations carried out in its support. The first, mentioned above (Paper I), contains a general introduction. The radio structure and astrometric stability of the reference sources are described in Papers II (Ransom et al. 2012a) and III (Bartel et al. 2012), respectively. The astrometric process and results are described in Paper IV (Lebach et al. 2012), Paper V (Ratner et al. 2012), and Paper VI (Ransom et al. 2012b). Early results on radio imaging and astrometry of IM Peg were presented in Ransom et al. (2005) and a brief overview was given in Bartel et al. (2008).

In this paper, we discuss the unprecedented sequence of VLBI images of IM Peg obtained as a result of our VLBI observations. These images, which have resolutions (east-west) typically about equal to the stellar angular radius, were obtained

\footnotetext{
3 Now also at Hartebeesthoek Radio Astronomy Observatory, PO Box 443, Krugersdorp 1740, South Africa.

4 Now at Okanagan College, 583 Duncan Avenue West, Penticton, BC V2A 2K8, Canada and also at the National Research Council of Canada, Herzberg Institute of Astrophysics, Dominion Radio Astrophysical Observatory, PO Box 248, Penticton, BC V2A 6K3, Canada.
}

in addition to the astrometry required for the $G P-B$ project. This set of images is the most extensive available for any radio star. ${ }^{5}$ RS CVn stars are known to have strong and relatively compact radio emission, and to be variable in radio flux density on timescales of an hour or even less (e.g., Lebach et al. 1999; Jones et al. 1996; Hjellming 1988; Dulk 1985; Mutel et al. 1985). For other VLBI images of RS CVn stars, we refer the interested reader to Ransom et al. (2002, 2003), Lestrade et al. (1995), Massi et al. (1988), and Mutel et al. (1985).

The guide star IM Peg is at a distance of $96.4 \pm 0.7 \mathrm{pc}$ (from VLBI parallax measurement; Hipparcos optical parallax measurements by ESA 1997 gave a consistent measure of this distance, albeit with rather larger uncertainties; see Paper V). The star has an orbital period of $\sim 25$ days. The primary is a K2 III star with a mass of $1.8 \pm 0.2 M_{\odot}$ and an effective temperature ranging from $\sim 3500$ to $5100 \mathrm{~K}$ (Berdyugina et al. 2000). It rotates rapidly, with $v \sin i \sim 27 \mathrm{~km} \mathrm{~s}^{-1}$ (Berdyugina et al. 2000), and its radius is $13.3 \pm 0.6 R_{\odot}$ (Berdyugina et al. $1999 b)$, so its angular diameter would be $1.28 \pm 0.06$ mas on the sky. The secondary, first spectroscopically detected by Marsden et al. (2005), has a mass of $\sim 1 M_{\odot}$ and an effective temperature of $\sim 5650 \mathrm{~K}$. The orbit has been accurately determined using optical spectroscopy, and here we use the parameters given in Marsden et al. (2005; see also Berdyugina et al. 1999b), namely, a period of $24.64877 \pm 0.00003$ days, with a (heliocentric) time for superior conjunction of the primary, with the primary at maximum distance from us, being the Julian date $2,450,342.905 \pm 0.004$ and an orbital eccentricity ${ }^{6}$ of 0 . The inclination angle of the orbit to the plane of the sky as determined

\footnotetext{
5 We note that Peterson et al. (2011) have made VLBI observations of the Algol and UX Arietis systems with slightly fewer epochs per source, but with coverage over longer spans of time.

6 Berdyugina \& Marsden (2006) suggest that the orbital eccentricity is 0.017 such a small deviation from 0 would not affect any of our conclusions.
} 
from our fit of the orbit to the positions determined by VLBI is $73^{\circ} \pm 8^{\circ}$ and the position angle (P.A.) of the ascending node is $41^{\circ} \pm 9^{\circ}$ (Paper V). This value is consistent with the earlier lower limit of $55^{\circ}$ set by Lebach et al. (1999), and is in good agreement with the range of $65^{\circ}$ and $80^{\circ}$ determined from optical observations (Berdyugina et al. 1999a).

In the optical, brightness variations of $\sim 0.3$ mag have been observed (Paper I; Ribárik et al. 2003; Strassmeier et al. 1997). Doppler imaging and photometry have shown that there are dark spots, which cover $\sim 15 \%$ of the star's surface, with temperatures more than $1500 \mathrm{~K}$ lower than the average. These spots persist over several orbital periods, although they seem to drift slowly on the surface of the star with respect to its orbital phase (Zellem et al. 2010; Berdyugina \& Marsden 2006; Ribárik et al. 2003; Berdyugina et al. 2000)

\section{OBSERVATIONS AND DATA REDUCTION}

The VLBI observations of IM Peg were carried out in 35 observing sessions between 1997 and 2005, using a global array of radio telescopes, at a frequency of $8.4 \mathrm{GHz}$. A fuller description of the VLBI observations as well as the basic data reduction is given in Paper II.

The National Radio Astronomy Observatory's phased Very Large Array (VLA) took part in 32 of the 35 sets of VLBI observations. By using the interferometric data from the VLA, we obtained accurate total flux-density measurements during the VLBI observations. We reduced the VLA data following standard procedures using NRAO's AIPS software package, with the amplitudes calibrated by using observations of the standard flux-density calibrators (3C 286 and 3C 48) and the scale of Baars et al. (1977). The flux densities were determined from images made from data which we self-calibrated in phase but not in amplitude. Since, for the majority of our observing sessions, the flux density varied significantly during the session, we list in Table 1 the maximum and minimum flux densities during each observing session as estimated from the light curves, along with the corresponding calendar date and Modified Julian date (MJD) of the midpoint of the observing session. We also list the fractional circular polarization, $m_{c}$. The circular polarization was calibrated by assuming that the calibrator sources have $m_{c}=0$ and by ignoring the leakage terms in the polarization response of the VLA antennas. The first of these assumptions is generally true to better than 1\% (Rayner et al. 2000), and the effect of the leakage terms at the VLA also has an effect of $<1 \%$ on the derived values of $m_{c}$. For the session of 2004 March 6, we performed a full polarization calibration and determined the leakage terms. We found that IM Peg had no detectable linear polarization.

Turning again to the VLBI data, we used phase-referenced astrometry, as described in Papers IV and V, to determine with unprecedented accuracy the proper motion of IM Peg. In particular, we estimated separately the star's secular proper motion, its projected orbit, and its parallax, all with respect to extragalactic reference sources. Knowledge of this proper motion and parallax allows us to place all the VLBI images of IM Peg in a star-centered frame. In particular, our solution for the overall motion of IM Peg allows us to estimate the position of the star's center for each observing session with a $1 \sigma$ uncertainty of $\lesssim 0.30$ mas in an extragalactic frame (Paper V).

Our final images were phase-referenced to a near-stationary feature in the brightness distribution of the quasar 3C 454.3. As the astrometry was expected to be critical for the success of the $G P-B$ project, it was done more elaborately than usual.
Table 1

Observing Sessions and Radio Flux Densities at $8.4 \mathrm{GHz}$

\begin{tabular}{|c|c|c|c|c|c|}
\hline \multirow[t]{2}{*}{ Date } & \multirow[t]{2}{*}{ MJD } & \multirow{2}{*}{$\begin{array}{l}\text { Orbital } \\
\text { Phase }^{\mathrm{a}}\end{array}$} & \multicolumn{2}{|c|}{ Total Flux Density ${ }^{b}$} & \multirow{2}{*}{$\begin{array}{l}\text { Fractional Circular } \\
\text { Polarization, } m_{c}{ }^{c} \\
(\%)\end{array}$} \\
\hline & & & $\begin{array}{l}\text { Minimum } \\
(\mathrm{mJy})\end{array}$ & $\begin{array}{c}\text { Maximum } \\
(\mathrm{mJy})\end{array}$ & \\
\hline 1997 Jan 16 & 50464.90 & 0.97 & 18 & 46 & $-0.5 \pm 2.0$ \\
\hline 1997 Jan 18 & 50466.89 & 0.05 & 6.8 & 21 & $-1.5 \pm 2.0$ \\
\hline 1997 Nov 30 & 50782.03 & 0.84 & 8.5 & 13 & $-1.4 \pm 2.0$ \\
\hline 1997 Dec 21 & 50803.96 & 0.73 & 48 & 76 & $-2.7 \pm 2.0$ \\
\hline 1997 Dec 27 & 50809.96 & 0.97 & 8.4 & 18 & $2.9 \pm 2.0$ \\
\hline 1998 Mar 1 & 50873.78 & 0.56 & 1.1 & 24 & $3.6 \pm 2.0$ \\
\hline 1998 Jul 12 & 51006.41 & 0.94 & 1.8 & 2.4 & $-3.7 \pm 2.0$ \\
\hline 1998 Aug 8 & 51033.35 & 0.03 & 2.9 & 16 & $1.9 \pm 2.0$ \\
\hline 1998 Sep 17 & 51073.24 & 0.65 & 11 & 28 & $3.6 \pm 2.0$ \\
\hline 1999 Mar 13 & 51250.74 & 0.85 & 0.7 & 3.3 & $0.9 \pm 2.0$ \\
\hline 1999 May 15 & 51313.57 & 0.40 & 2.0 & 7.3 & $4.7 \pm 2.0$ \\
\hline 1999 Sep 19 & 51440.23 & 0.54 & 12 & 25 & $-0.9 \pm 2.0$ \\
\hline 1999 Dec 9 & 51521.99 & 0.86 & 2.1 & 3.8 & $1.2 \pm 2.0$ \\
\hline 2000 May 15 & 51679.56 & 0.25 & 0.2 & 0.9 & $12.3 \pm 3.7$ \\
\hline 2000 Aug 7 & 51763.34 & 0.65 & 8.0 & 58 & $0.3 \pm 2.0$ \\
\hline 2000 Nov 6 & 51854.09 & 0.33 & 0.6 & 5.7 & $2.0 \pm 2.0$ \\
\hline 2000 Nov 7 & 51855.01 & 0.37 & 8.4 & 10.6 & $-0.5 \pm 2.0$ \\
\hline 2001 Mar 31 & 51999.73 & 0.24 & 0.3 & 0.3 & $10.4 \pm 9.7$ \\
\hline 2001 Jun 29 & 52089.48 & 0.88 & 0.4 & 1.0 & $-20.1 \pm 4.0$ \\
\hline 2001 Oct 20 & 52202.05 & 0.45 & 4.2 & 8.1 & $-4.3 \pm 2.0$ \\
\hline 2001 Dec 21 & 52264.99 & 1.00 & 1.2 & 1.2 & $5.5 \pm 2.1$ \\
\hline 2002 Apr 14 & 52378.65 & 0.61 & 0.3 & 0.6 & $16.3 \pm 3.6$ \\
\hline 2002 Jul 14 & 52469.40 & 0.29 & $0.47^{\mathrm{d}}$ & 0.47 & \\
\hline 2002 Nov 21 & 52599.06 & 0.55 & $0.32^{\mathrm{d}}$ & 0.32 & \\
\hline 2003 Jan 26 & 52665.88 & 0.26 & 0.20 & 0.28 & $1.0 \pm 5.8$ \\
\hline 2003 May 18 & 52777.55 & 0.79 & 1.0 & 1.1 & $15.0 \pm 5.0$ \\
\hline 2003 Sep 9 & 52891.24 & 0.41 & 0.5 & 0.7 & $13.3 \pm 3.2$ \\
\hline 2003 Dec 6 & 52979.00 & 0.97 & $0.9^{\mathrm{d}}$ & 0.9 & \\
\hline 2004 Mar 6 & 53070.76 & 0.69 & 8.0 & 19 & $-0.5 \pm 2.0$ \\
\hline 2004 May 18 & 53143.58 & 0.64 & 10 & 12 & $-1.4 \pm 2.0$ \\
\hline 2004 Jun 26 & 53182.49 & 0.22 & 5.0 & 12 & $0.9 \pm 2.0$ \\
\hline 2004 Dec 12 & 53351.00 & 0.06 & 0.7 & 1.1 & $-6.3 \pm 2.9$ \\
\hline 2005 Jan 15 & 53385.92 & 0.48 & 0.25 & 0.35 & $44.3 \pm 5.9$ \\
\hline 2005 May 28 & 53518.45 & 0.86 & 0.25 & 0.85 & $27.9 \pm 5.5$ \\
\hline 2005 Jul 16 & 53567.41 & 0.84 & 0.24 & 0.36 & $44.6 \pm 7.7$ \\
\hline
\end{tabular}

Notes.

a The orbital phase for the midpoint of our observing session, in fractions of an orbit period, as determined from the orbit of Marsden et al. (2005).

${ }^{\mathrm{b}}$ Minimum and maximum total radio flux densities during the observing session, estimated from the light curves from concurrent VLA measurements, after boxcar smoothing with a window about 20 minute wide, except for the noted sessions where the flux densities were estimated from the VLBI measurements. The measurement uncertainties in the flux densities are usually dominated by the systematic uncertainty in the flux-density calibration which we take to be $5 \%$ for the VLA and $10 \%$ for VLBI. We do not list the uncertainties because the intrinsic variability of IM Peg on short time scales is generally larger than the measurement uncertainty.

c Average of the fractional circular polarization, $m_{c}$ (labeled FCP; IEEE convention), during the observing session, in $\%$. We adopt a minimum $1 \sigma$ uncertainty in the circular polarization of $2 \%$.

${ }^{\mathrm{d}}$ Flux densities determined from VLBI measurements.

We used both phase-referenced mapping and parametric model fits to the measured fringe phases using a Kalman filter. The fringe and phase calibration used for the final images in this paper is the same as was used to obtain our final astrometric results, and involved a combination of both these methods. The two methods and their relative advantages, as well as a new combination of the two, are described in Paper IV. We carried out amplitude calibration in AIPS, using CLEAN models of 
the calibrator sources, 3C 474.3, B2250+194, and B2252+172 (see Papers II and III). The resulting phase-referenced images of IM Peg should represent a relatively unbiased estimate of the source brightness distribution, since the calibration was derived without any reference to the IM Peg visibilities. In particular, no spurious symmetrization should have occurred, as often results from self-calibration that is based on an initial point-source model (see Massi \& Aaron 1999), nor should any reduction of the circular polarization have taken place, as can occur when one performs amplitude self-calibration. We note that as with the VLA data above, we have assumed that our calibrator sources had $m_{c}=0$, which is unlikely to introduce errors of $>1 \%$ in our circular polarization images of IM Peg. Furthermore, any motion of the radio emission during the course of an observing session has not been significantly suppressed by self-calibration in phase.

\section{RESULTS}

\subsection{Total Flux Densities}

We determined the total radio flux density of IM Peg during each of our observing sessions, in most cases from VLA observations. IM Peg's flux density varied detectably during almost all observing sessions, with variations on timescales as short as $\sim 30$ minutes, and over a range in flux density exceeding 30:1 in a single session (and overall exceeding 300:1). We tabulate the maximum and minimum flux densities for each session in Table 1, and show four example light curves in Figure 1.

Significant circular polarization, also generally time variable, was observed in many cases, and we list as a percentage the average circular polarization, defined as $(R-L) /(R+L)$, for each observing session in Table 1 . We defer discussion of the circular polarization to Section 3.4 below.

\subsection{VLBI Images}

In Figure 2, we present the $8.4 \mathrm{GHz}$ VLBI images of IM Peg for all 35 observing sessions, while Table 2 lists some characteristics of the images including their peak and rms background brightness values. As shown in Table 1, IM Peg's total flux density, as monitored by VLA observations, varied significantly during a number of our VLBI observing sessions. In such cases, the VLBI image made from the whole data set (such as those in Figure 2) represents the average emission over the observing session, albeit with some possible distortion due to the incomplete removal of sidelobes. ${ }^{7}$ The rms background brightness values, given in Table 2, are estimated from empty regions in each image. They represent a lower bound on the image brightness uncertainty. Adding to this bound are the effects of the aforementioned distortion due to time variability and deconvolution errors, which limit the image fidelity (see, e.g., Briggs et al. 1999 and Briggs 1995; see also discussion in Bietenholz et al. 2003). The images were made by first weighting the visibilities with the inverse square root of the

\footnotetext{
7 Images made from earth-rotation aperture synthesis observations, such as our VLBI images, are generally based on the assumption that the source brightness distribution does not change during the observations. Since both the brightness distribution and the instantaneous point-spread function are time variable, and since deconvolution is a nonlinear process, the deconvolved image does not strictly represent the time-averaged brightness distribution. However, provided that the change in the brightness distribution is not gross and that the sidelobes are relatively low compared to the peak of the point-spread function, a deconvolved image should be a reasonable approximation to the time-averaged brightness distribution.
}

nominal statistical variance in the visibility measurements ${ }^{8}$ and then further modifying the weighting by the robust weighting scheme of Briggs (1995) as implemented in AIPS.

We display the VLBI images in Figure 2, centered on the chromospherically active primary based on our astrometry, which includes fits to the $\sim 25$ day orbit and the parallax, as well as the secular proper motion of the star (see Papers IV, V, and VI). This identification of the star's center in each image is based not only on our astrometry, but also on the assumption that the radio emission is, on average, centered on the star. It is possible, although we consider it less likely, that there is a significant systematic offset of the radio emission from the center of the star. The rms scatter in position over all observing sessions, based on the residuals, is $\sim 0.4$ mas in both $\alpha$ and $\delta$. This scatter is dominated by the variability in the position of the radio source with respect to the star's center, as our astrometric uncertainty is $<0.14$ mas. We expect our fit position for the star's center, which we use as the origin in each panel of Figure 2, to be in error by no more than 0.3 mas $(1 \sigma)$, with the likely errors being smaller (see Paper V).

\subsection{Rapid Time Evolution of the Images from VLBI}

The $u-v$ coverage of our array is dense enough to allow us to produce images from subsets of our data for each observing session, each covering several consecutive hours. As two examples, we show in Figure 3 images made by partitioning the data from the observing sessions of 1997 December 27 and 1999 September 19, respectively, into three time ranges. The time ranges were chosen so as to have an approximately equal number of visibility measurements in each interval.

Unfortunately, as the $u-v$ coverage changes, so does the elliptical convolving beam. Convolving with a common, round beam would have allowed easier comparison of the three time ranges, but at the expense of losing much of the image structure to the resulting lower resolution.

Both these observing sessions show similar temporal behavior. In the first two of the three time ranges, the source exhibits a double structure very similar to that of the average image shown in Figure 2, with two distinct brightness maxima. In both sessions, in the last of the three time ranges, the effective resolution is lower, and it is no longer possible to distinguish the two maxima, although the presence of a double structure similar to that seen in the first two time ranges is compatible with the image.

In both examples, the double structure seems to persist over most or all of our $\sim 12 \mathrm{hr}$ observing sessions. We can conclude that the double structure is not an artifact of motion over short timescales. Such rapid motion was in fact seen on 1997 January 16 (Lebach et al. 1999). Smaller motions on hour timescales occurred in our 1998 March 1 and 1998 August 8 sessions, both of which show strong flux-density variability (see Ransom et al. 2005). These rapid motions are much faster than the orbital motion or that due to rotation of the primary star,

\footnotetext{
8 The nominal statistical variance of the visibility measurements reflects the uncertainties due only to random measurement noise. If such random noise were the only source of error in the visibility measurements, then the most efficient weighting would be weighting by the inverse of the nominal variance However, in our case, especially for the more sensitive telescopes, the effective uncertainty in a particular visibility measurement can be dominated by small residual calibration errors rather than statistical noise. These calibration errors will depend only weakly on the telescope sensitivity. For this reason, we weighted by the inverse square root of the variance. This compresses the weights, and although ad hoc, should lead to a more robust image at the possible expense of a slight loss of signal-to-noise ratio.
} 

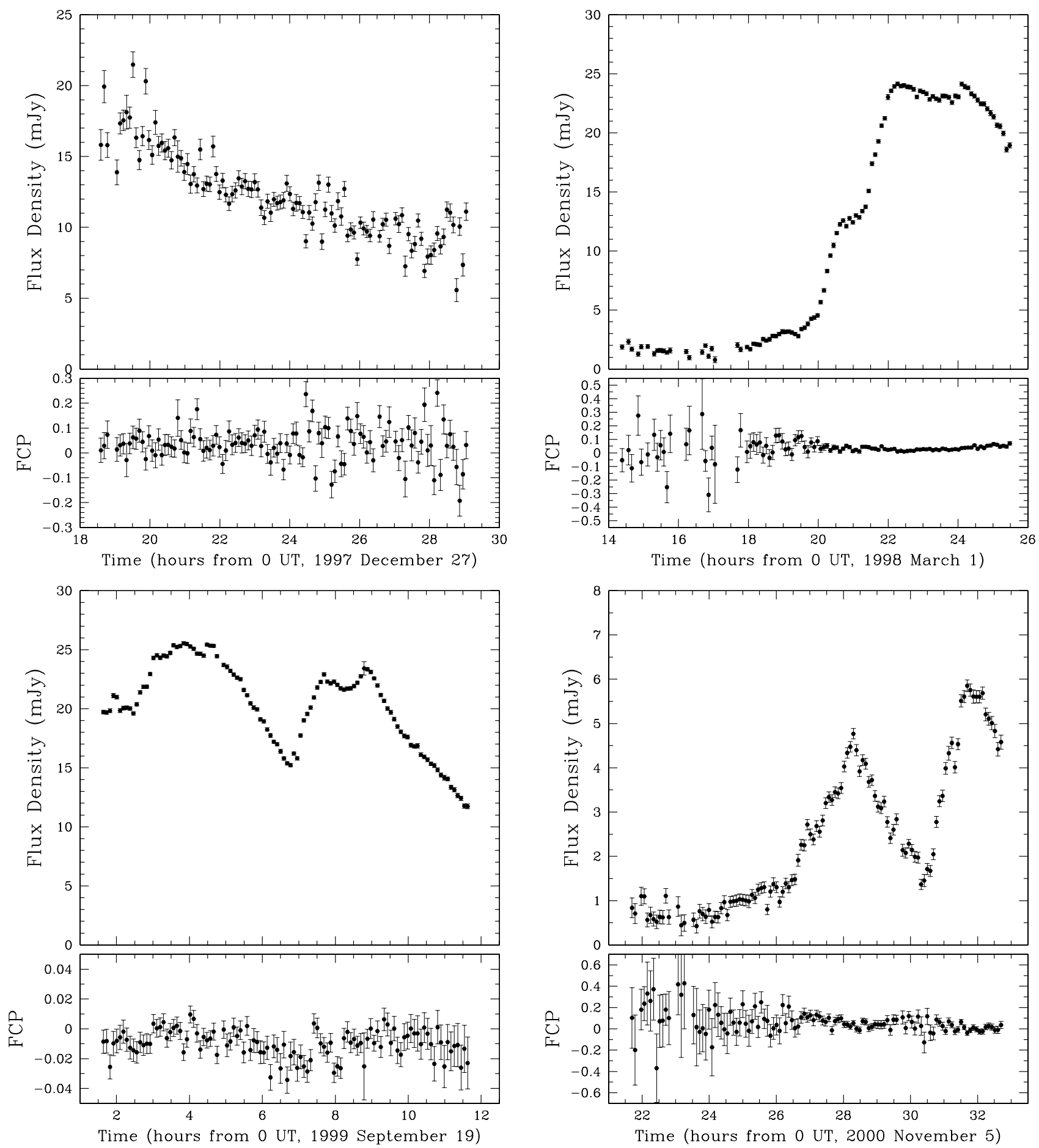

Figure 1. Total $8.4 \mathrm{GHz}$ flux density (top curves) and fractional circular polarization, $m_{c}$ (IEEE convention; lower curves), of IM Peg as functions of time, as determined from VLA observations, shown for four example observing sessions, namely, 1997 December 27, 1998 March 1, 1999 September 19, and 2000 November 6. Note that the dates given in horizontal axis labels in this figure are start dates, so that the lower right panel refers to the observing session with the midpoint date of 2000 November 6 in Table 1. The plotted $1 \sigma$ uncertainties are statistical only and do not include an estimated 5\% systematic uncertainty in the VLA flux-density calibration. (Within each session, any calibration error in the flux density is not expected to vary rapidly as a function of time.)

which are expected to be only $\sim 0.03$ mas over $3 \mathrm{hr}$, and thus not easily detectable in a VLBI session.

\subsection{Polarization}

For the 2004 March 6 epoch, for which we performed a full polarization calibration, we measured a linear polarization fraction for IM Peg of $<1 \%$. This is consistent with the expectation that Faraday rotation within the corona of an
RS CVn system will substantially depolarize any centimeterwavelength radio emission (Paredes 2005). We do not discuss linear polarization further.

RS CVn systems are, on the other hand, expected to show substantial circular polarization due to the gyrosynchrotron mechanism. We did therefore determine the average circular polarization for each observing session from our VLA data (see Table 1). We plot the measured values of the fractional circular polarization, $m_{c}$, in the left panel of Figure 4 . The maximum 

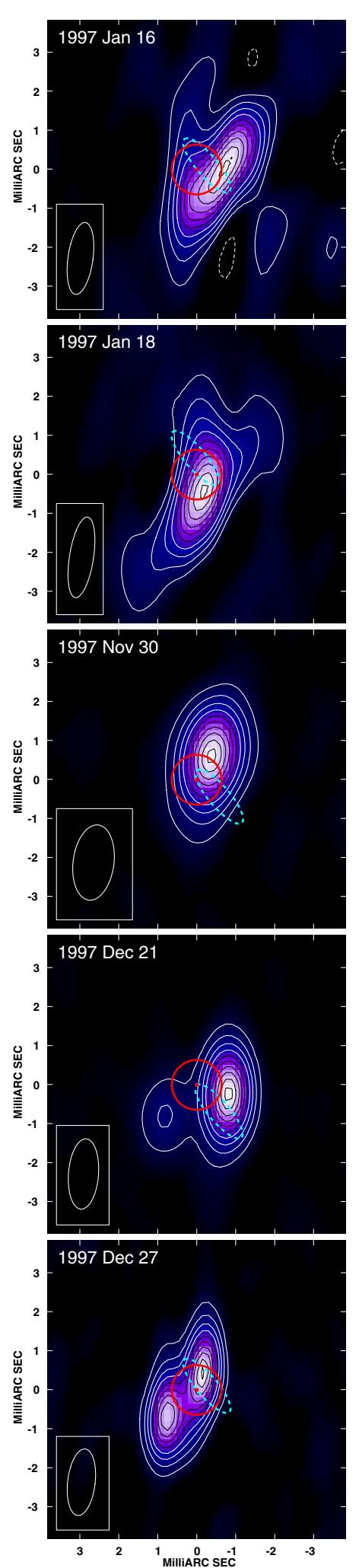
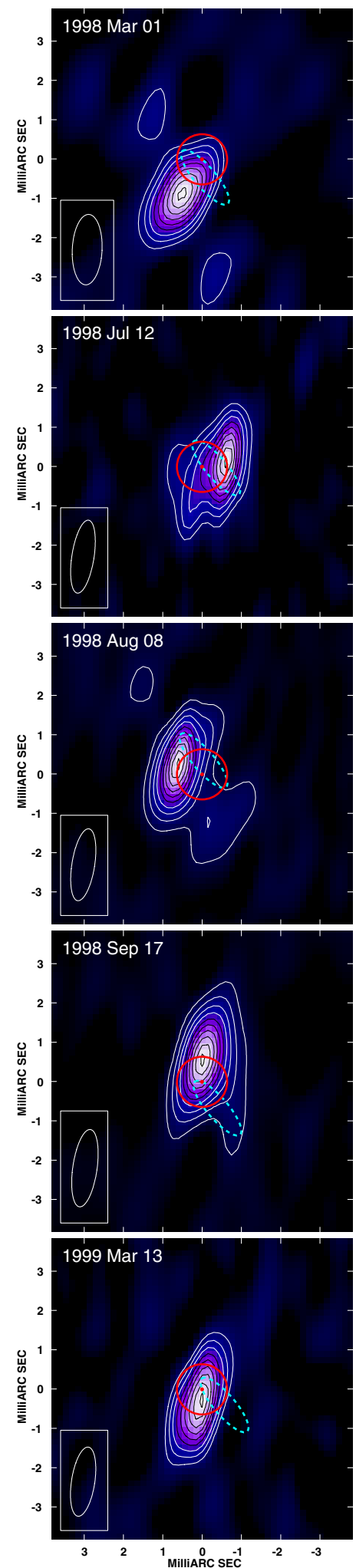
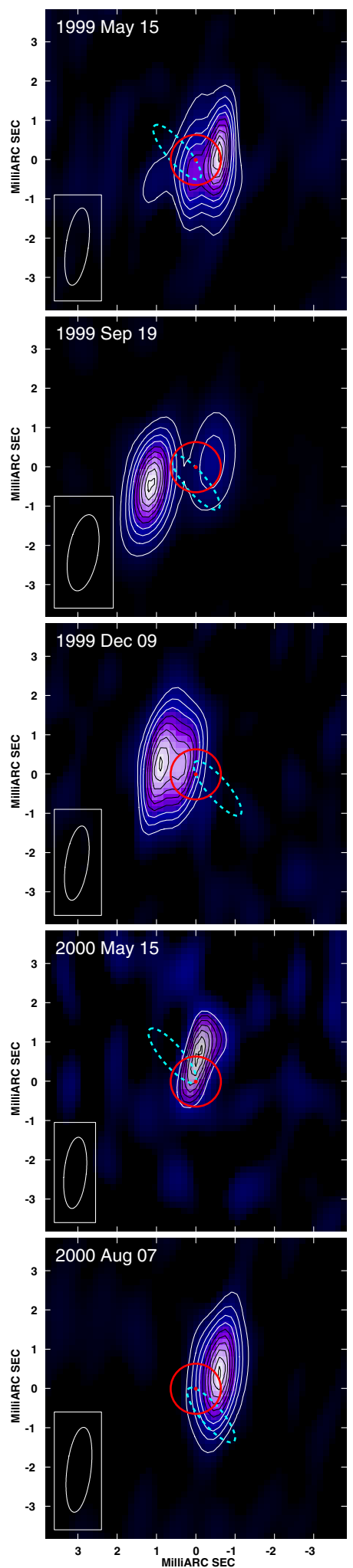

Figure 2. VLBI images of IM Peg at $8.4 \mathrm{GHz}$. The observing date is indicated in the top left of each panel, while the FWHM of the convolving beam is indicated in the lower left. North is up and east is to the left. Both the contours and the color scale show the brightness. The contours are drawn at $10 \%, 20 \%, 30 \%, \ldots$, $80 \%, 90 \%$, and $98 \%$ of the peak brightness, starting with the first contour above three times the rms background; and contours at 50\% and above are drawn in black. The peak brightness and rms background values are listed in Table 2. The center of each panel (red dot) is the fit position of the star's center, as derived from the astrometric results of Paper V. In other words, the coordinate origin in our radio image should approximately represent the center of the disk of the primary star. The red circle indicates the angular size of the primary star (radius of $13.3 \pm 0.6 R_{\odot}$; Berdyugina et al. 1999b). The cyan dotted ellipse shows the binary orbit of the primary (Paper V). Note that we have chosen to keep the primary star, rather than the center of the binary orbit at the center of our plots. We also include an mpeg animation showing the evolution of IM Peg's radio emission. This animation consists of a simple linear interpolation in brightness between consecutive observing sessions. It is intended to be illustrative only, as our sampling is not rapid enough to follow the evolution of the radio emission in detail. In the animation that accompanies this figure, color is used to indicate the total flux density at each epoch, with blue indicating low flux densities and redder colors indicating higher total flux densities. Time is linear, with each frame in the animation corresponding to approximately four days.

(An animation of this figure is available in the online journal.) 

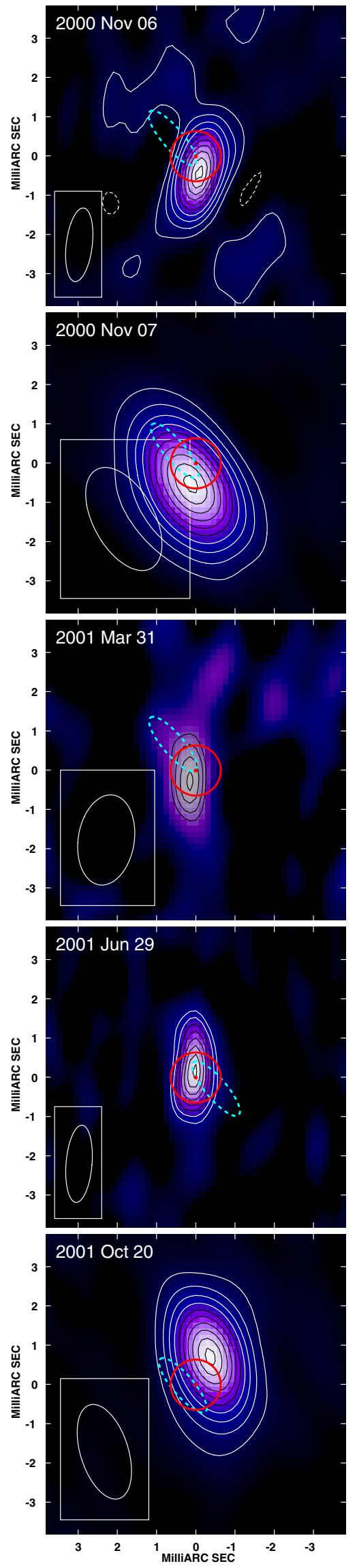
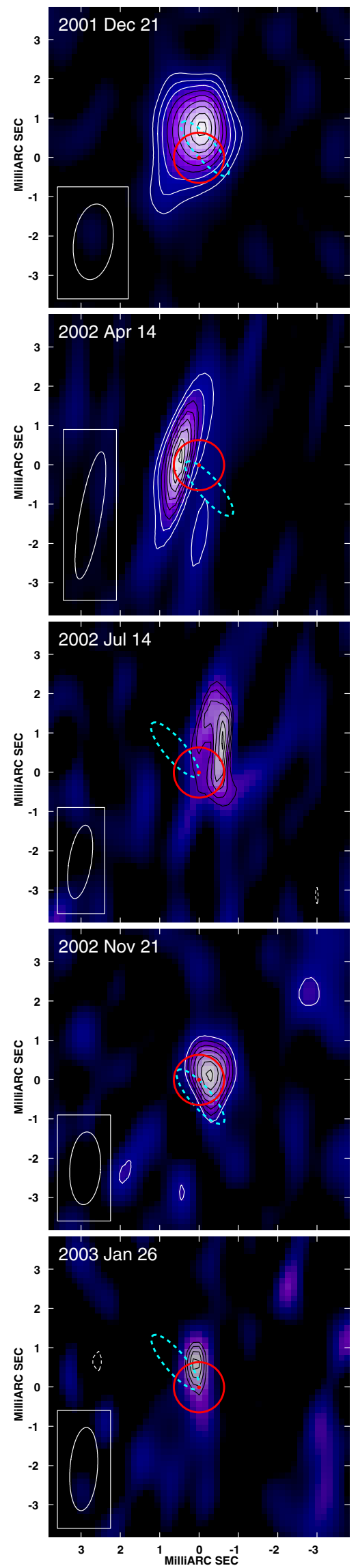

Figure 2. (Continued)
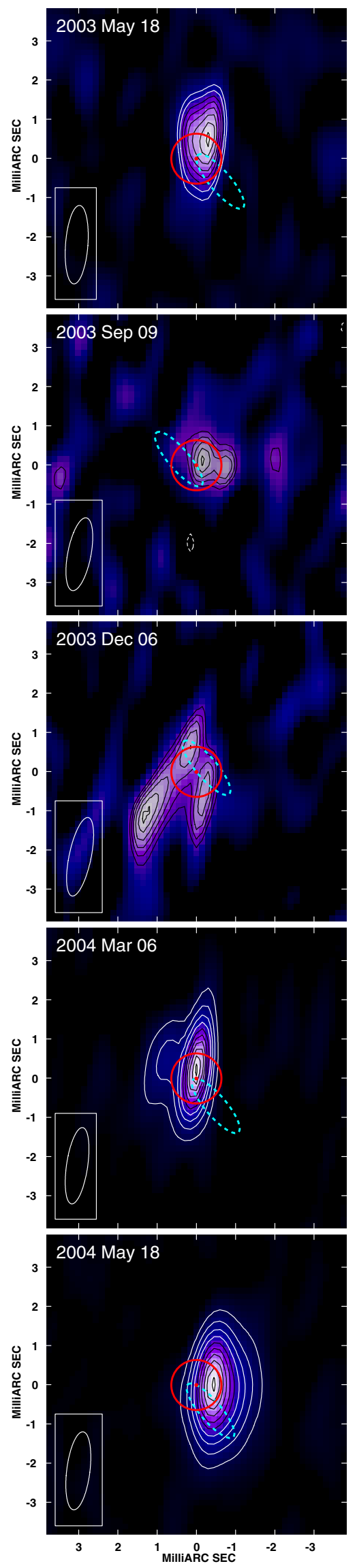

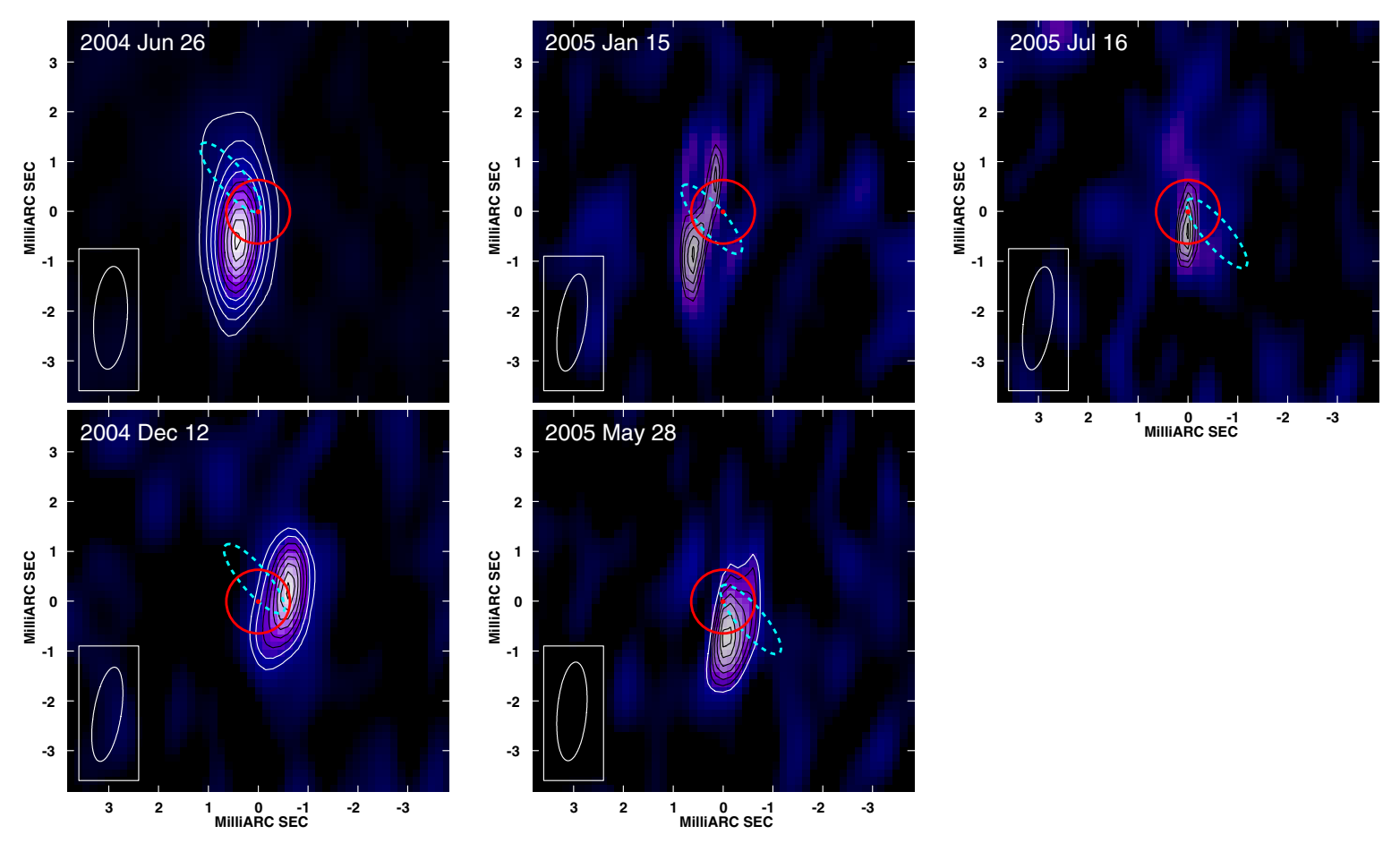

Figure 2. (Continued)

observed value of $\left|m_{c}\right|$ was $\sim 46 \%$ (on 2005 July 16). Values of $\left|m_{c}\right|>10 \%$ were only seen when the flux density was below 2 mJy. Higher values of $\left|m_{c}\right|$ are observed after 2004, when the total flux density was generally lower, suggesting that $\left|m_{c}\right|$ is anticorrelated with the total flux density.

Over the 32 observing sessions for which we had VLA data, the weighted average of $m_{c}$ was $1.3 \% \pm 0.4 \%$, with the standard deviation being $13 \%$, and the cited uncertainty being statistical only. This result suggests a marginally significant $(3.1 \sigma)$ average positive circular polarization. However, an additional systematic error, which we estimate at $1 \%$, must be added because of the possible deviations from the assumed value of zero for $m_{c}$ of our calibrator sources as well as the uncorrected leakage terms. The average value of $m_{c}$ therefore cannot be regarded as significantly different from 0 .

We also found no obvious correlation of $m_{c}$ with orbital phase. We plot the fractional circular polarization against orbital phase in the right panel of Figure 4; no obvious correlation with orbital phase is observed. Note that here and throughout this paper, we calculate the orbital phase at the midpoint of each observing session, in fractions of an orbit period, as determined from the orbit of Marsden et al. (2005).

Although, as noted, the average value of $m_{c}$ was not significantly different from 0 , we did find evidence for a significantly positive value of $m_{c}$ when the flux density was low. We examine only those epochs which had a flux density of $<2 \mathrm{mJy}$ and found that the weighted average $m_{c}$ was $4.3 \% \pm 0.9 \%$. Of these epochs, 10 out of 12 have positive $m_{c}$. The chance probability of observing this many or more positive values of $m_{c}$ if the sign were random is $1.9 \%$. We conclude that for low flux densities, there is a high probability that IM Peg is right (IEEE convention) circularly polarized.

Significant circular polarization is commonly detected in RS CVn stars. IM Peg seems to follow the trends observed for other systems, in that $m_{c}$ has a somewhat consistent sign, but the magnitude decreases with increasing flux density. It has been proposed (e.g., Lestrade et al. 1988; Mutel et al. 1985) that this pattern is due to flares radiating via the gyrosynchrotron mechanism. Each flare represents some release of energy. Shortly after the initial release, the emission is optically thick, hence of high brightness but of low circular polarization. As the flare decays, the brightness as well as the optical thickness decreases, and the fractional circular polarization increases. The low-level "quiescent" emission might then just be the superposition of the decays of many small flare events. Note that an alternative explanation for the quiescent emission which has been proposed is that it represents gyrosynchrotron emission from a thermal (Maxwellian) population of electrons (e.g., Drake et al. 1989). Arguments against this hypothesis are given in Beasley \& Güdel (2000) and Paredes (2005). Our observation of high circular polarization when IM Peg's flux density was low, as well as our observation of large source sizes, also when IM Peg was quite weak (e.g., during the observing session of 2005 May 15) argues further against this hypothesis. We therefore think that the quiescent radio emission is due to a non-thermal, rather than a thermal, population of electrons.

For our VLBI observing sessions, we recorded both right and left circular polarizations, and can therefore image the circularly polarized flux density. Figure 5 shows three example images. The distribution of circular polarization clearly varies from session to session. Some sessions, such as that of 1998 September 17, show a fractional circular polarization that is approximately constant across the emission region; for other sessions, for example 2004 March 6, there are prominent gradients.

\subsection{Parameterization of the Emission Geometry}

To investigate the temporal evolution of the emission geometry, we sought to parameterize it. As a simple first approach fitted an elliptical Gaussian to the image. Although such a fit does not adequately describe the complexity of the emission region, especially when the emission region shows a double structure, the fitted major axis and its P.A. nonetheless give an indication of the overall extension and the direction of elongation 
Table 2

Image Characteristics

\begin{tabular}{|c|c|c|c|c|c|c|}
\hline $\begin{array}{l}\text { Date } \\
\text { (midpoint) }\end{array}$ & $\begin{array}{c}\text { MJD } \\
\text { (midpoint) }\end{array}$ & $\begin{array}{l}\text { Peak Brightness } \\
\quad\left(\mathrm{mJy} \mathrm{bm}^{-1}\right)\end{array}$ & $\begin{array}{l}\text { Image RMS } \\
\left(\mathrm{mJy} \mathrm{bm}^{-1}\right)\end{array}$ & $\begin{array}{l}\text { Major Axis }{ }^{\mathrm{b}, \mathrm{e}} \\
\text { (mas) }\end{array}$ & Axis Ratio $^{\mathrm{c}, \mathrm{e}}$ & $\begin{array}{l}\text { P.A. }{ }^{\mathrm{d}, \mathrm{e}} \\
\quad\left({ }^{\circ}\right)\end{array}$ \\
\hline 1997 Jan 16 & 50464.90 & 7.97 & 0.15 & 2.78 & 0.22 & 136 \\
\hline 1997 Jan 18 & 50466.89 & 4.67 & 0.07 & 1.93 & 0.40 & 149 \\
\hline 1997 Nov 30 & 50782.03 & 6.85 & 0.06 & 1.00 & 0.57 & 140 \\
\hline 1997 Dec 21 & 50803.96 & 31.9 & 0.15 & 0.77 & 0.79 & 13 \\
\hline 1997 Dec 27 & 50809.96 & 4.61 & 0.06 & 2.18 & 0.27 & 146 \\
\hline 1998 Mar 1 & 50873.78 & 5.52 & 0.22 & 1.49 & 0.00 & 124 \\
\hline 1998 Jul 12 & 51006.41 & 0.72 & 0.04 & 1.62 & 0.50 & 134 \\
\hline 1998 Aug 8 & 51033.35 & 4.03 & 0.06 & 0.89 & 0.46 & 130 \\
\hline 1998 Sep 17 & 51073.24 & 12.6 & 0.13 & 0.99 & 0.46 & 127 \\
\hline 1999 Mar 13 & 51250.74 & 0.92 & 0.04 & 1.64 & 0.35 & 153 \\
\hline 1999 May 15 & 51313.57 & 2.09 & 0.04 & 0.98 & 0.48 & 105 \\
\hline 1999 Sep 19 & 51440.23 & 13.1 & 0.10 & 1.38 & 0.75 & 170 \\
\hline 1999 Dec 9 & 51521.99 & 1.05 & 0.04 & 0.29 & 0.52 & 93 \\
\hline 2000 May 15 & 51679.56 & 0.39 & 0.04 & 1.43 & 0.15 & 149 \\
\hline 2000 Aug 7 & 51763.34 & 32.7 & 0.43 & 0.53 & 0.62 & 109 \\
\hline 2000 Nov 6 & 51854.09 & 1.87 & 0.05 & 1.01 & 0.00 & 125 \\
\hline 2000 Nov 7 & 51855.01 & 6.82 & 0.12 & 0.89 & 0.30 & 97 \\
\hline 2001 Mar 31 & 51999.73 & 0.23 & 0.05 & 2.53 & 0.00 & 10 \\
\hline 2001 Jun 29 & 52089.48 & 0.44 & 0.03 & 1.09 & 0.48 & 16 \\
\hline 2001 Oct 20 & 52202.05 & 5.17 & 0.07 & 0.95 & 0.57 & 134 \\
\hline 2001 Dec 21 & 52264.99 & 0.78 & 0.05 & 1.12 & 0.93 & 92 \\
\hline 2002 Apr 14 & 52378.65 & 0.36 & 0.03 & 0.69 & 0.00 & 3 \\
\hline 2002 Jul 14 & 52469.40 & 0.24 & 0.04 & 2.74 & 0.29 & 1 \\
\hline 2002 Nov 21 & 52599.06 & 0.29 & 0.05 & 0.74 & 0.00 & 162 \\
\hline 2003 Jan 26 & 52665.88 & 0.21 & 0.05 & 0.68 & 0.00 & 21 \\
\hline 2003 May 18 & 52777.55 & 0.52 & 0.04 & 0.84 & 0.85 & 160 \\
\hline 2003 Sep 9 & 52891.24 & 0.24 & 0.05 & 1.46 & 0.00 & 69 \\
\hline 2003 Dec 6 & 52979.00 & 0.27 & 0.05 & 2.41 & 0.51 & 136 \\
\hline 2004 Mar 6 & 53070.76 & 9.52 & 0.09 & 0.71 & 0.54 & 179 \\
\hline 2004 May 18 & 53143.58 & 5.28 & 0.06 & 1.02 & 0.75 & 28 \\
\hline 2004 Jun 26 & 53182.49 & 4.77 & 0.06 & 1.13 & 0.42 & 4 \\
\hline 2004 Dec 12 & 53351.00 & 0.53 & 0.04 & 1.20 & 0.65 & 177 \\
\hline 2005 Jan 15 & 53385.92 & 0.14 & 0.02 & 3.3 & 0.22 & 175 \\
\hline 2005 May 28 & 53518.45 & 0.29 & 0.03 & 1.36 & 0.28 & 152 \\
\hline 2005 Jul 16 & 53567.41 & 0.13 & 0.03 & 1.50 & 0.00 & 20 \\
\hline
\end{tabular}

Notes.

a The rms background as estimated from empty regions in each image; this rms represents a lower limit to the image uncertainty (see the text)

b The FWHM major axis, $b$, of an elliptical Gaussian fitted to the image.

$\mathrm{c}$ The axis ratio of an elliptical Gaussian fitted to the image.

d The P.A. of the major axis, $\theta_{b}$, of an elliptical Gaussian fitted to the image, constrained to be $<180^{\circ}$.

e The values are those for an elliptical Gaussian which is the deconvolution of the (elliptical Gaussian) restoring beam from the elliptical Gaussian fitted to the CLEAN image by least squares.

of the emission region. We denote the vector major axis of the fitted Gaussian by $\mathbf{b}$ and its p.a. by $\theta_{b}$. Note that to avoid the bias introduced by convolution with the CLEAN beam, we do not use directly the values from the elliptical Gaussian fitted to the image, but rather those from the "deconvolved" elliptical Gaussian. The deconvolved elliptical Gaussian is the one which, when convolved with the elliptical Gaussian clean beam, results in the fitted elliptical Gaussian. The "deconvolved" fitted values of $|\mathbf{b}|, \theta_{b}$, and the axis ratio are given in Table 2 for each session. We also plot $\mathbf{b}$ on the projection of the binary orbit in Figure 6.

Figure 6 shows that the values of $\theta_{b}$ are not randomly distributed, but rather show a preferred orientation. The average ${ }^{9}$ of $\mathbf{b}$ was 0.71 mas along $\theta_{b}=157^{\circ}$. A bootstrap calculation gives

\footnotetext{
9 Note that the quantities $\mathbf{b}$ are axial, rather than true, vectors in that $\theta_{b}=90^{\circ}$ is equivalent to $\theta_{b}=-90^{\circ}$. In order to meaningfully average $\mathbf{b}$, we follow Batschelet (1981) and first double the P.A.'s $\left(\theta_{b}\right)$, then perform the usual vector average, and then halve the P.A. of the result to obtain the final average value of $\mathbf{b}$.
}

a statistical uncertainty of $7^{\circ}$ for the average direction of $\mathbf{b}$; however, systematic uncertainties, for example stemming from the "deconvolution" of the clean beam, are likely to be several times larger. The probability ${ }^{10}$ of $n=35$ random values being so well aligned is $\lesssim 1 \%$. We note that the above calculation used a P.A. derived from a single elliptical Gaussian fit to the entire emission region.

\section{DISCUSSION}

Using VLBI, we have produced a series of high-quality images of the radio emission from the RS CVn star, IM Peg. Our east-west resolution is approximately equal to the stellar radius of the primary; our north-south resolution is $\sim 3$ times poorer. The images show that the radio emission exhibits a

\footnotetext{
10 The chance probability was estimated from a 5000-trial Monte Carlo simulation, with each trial using 35 vectors with the same lengths as the measured values of $\mathbf{b}$, but with random orientations between $0^{\circ}$ and $180^{\circ}$.
} 

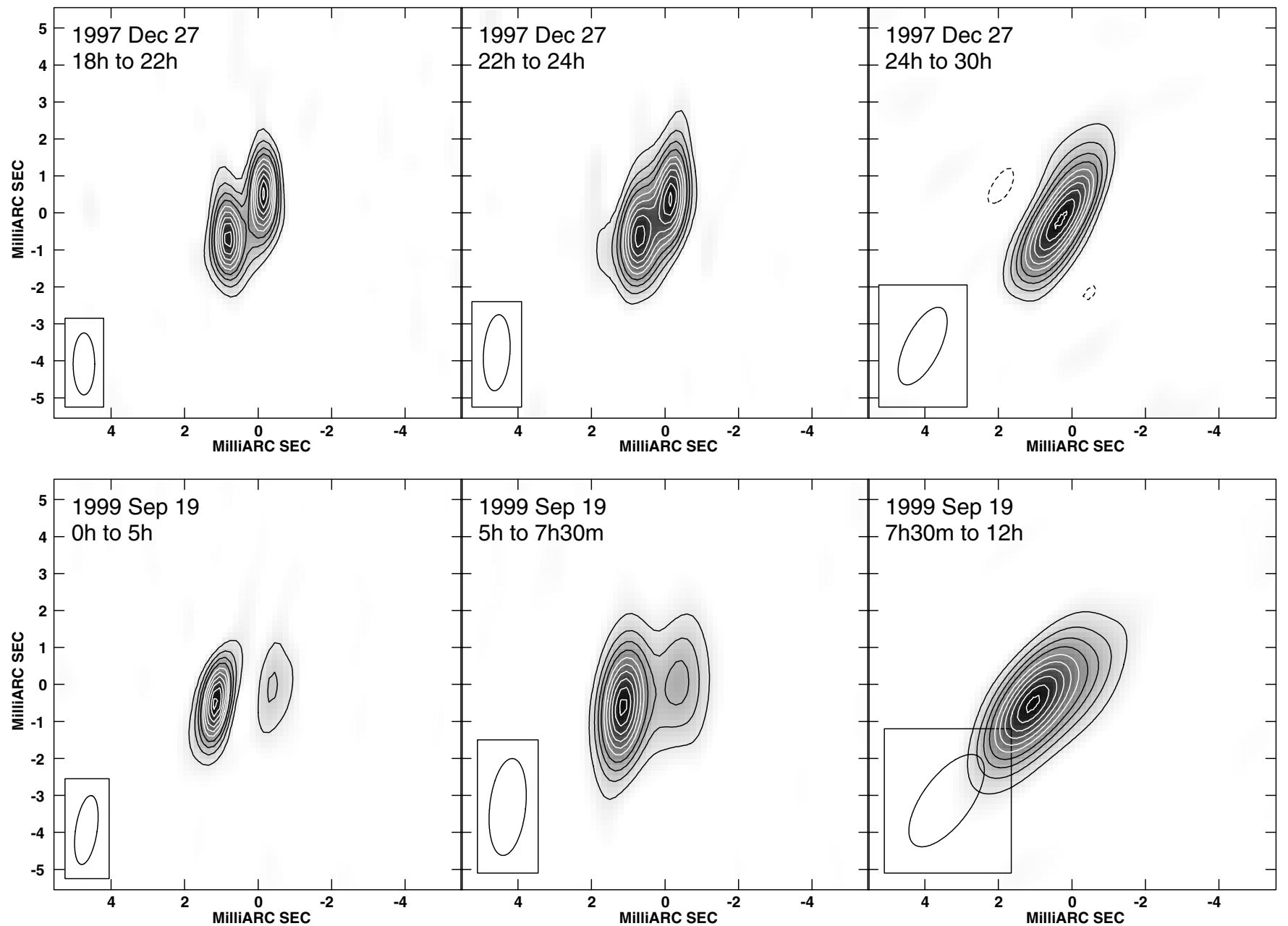

Figure 3. Time-resolved images of IM Peg on 1997 December 27 (top row) and 1999 September 19 (bottom row). In each row, the three panels show the VLBI images in the three consecutive UT time ranges given. The convolving beam, which varies with time, is indicated at lower left in each panel. Contours and gray scale are similar to those in Figure 2 . The three time ranges were chosen so that each has an approximately equal number of visibility measurements. The corresponding light curves were given in Figure 1.
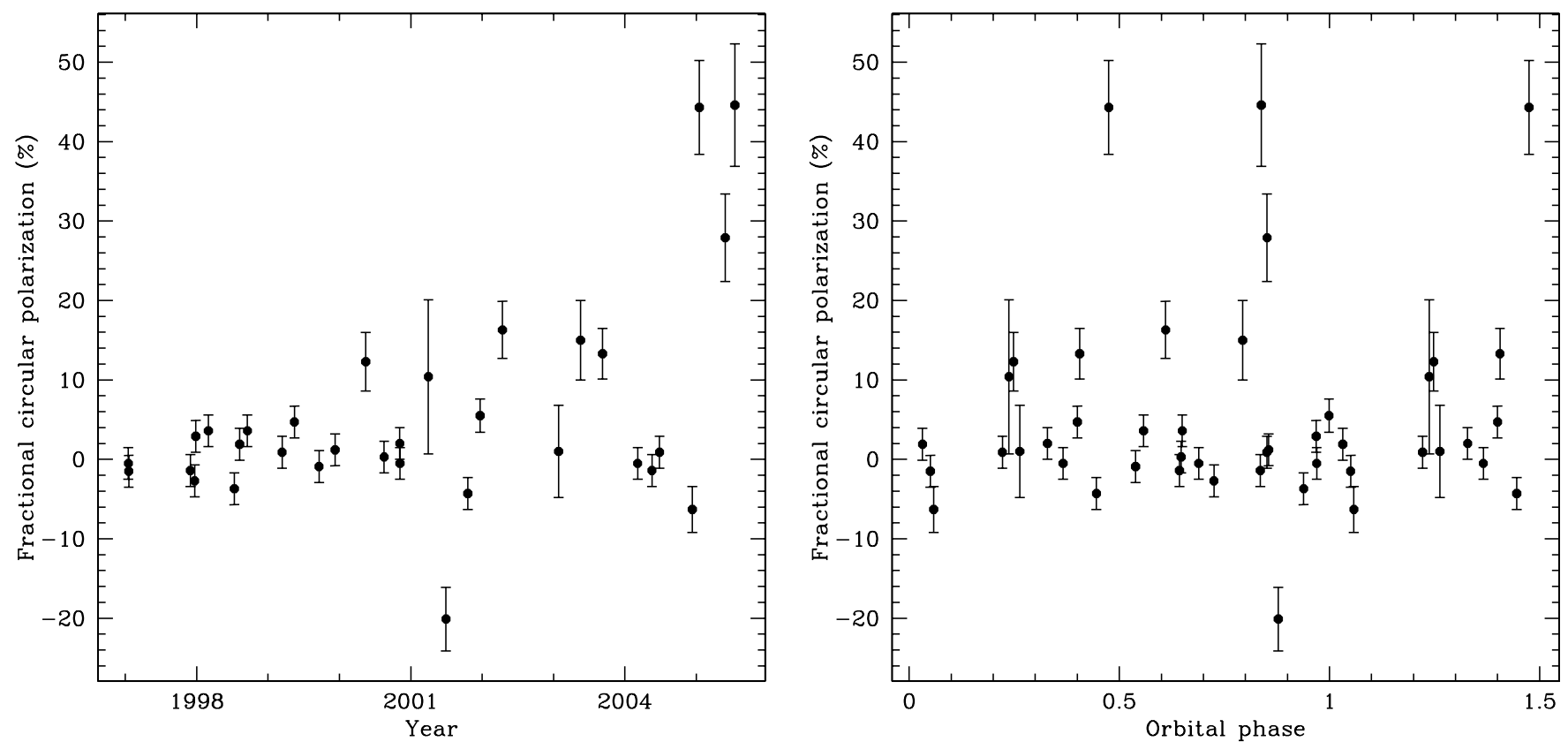

Figure 4. Left panel: fractional circular polarization, $m_{c}$ (IEEE convention), as determined from our VLA observations, plotted against year. Right panel: $m_{c}$ plotted against orbital phase (using the orbit of Marsden et al. 2005). Points with orbital phases between 0 and 0.5 are repeated on the right of the plot (with phases between 1.0 and 1.5 ) to make any possible cyclical variation more clearly visible. 

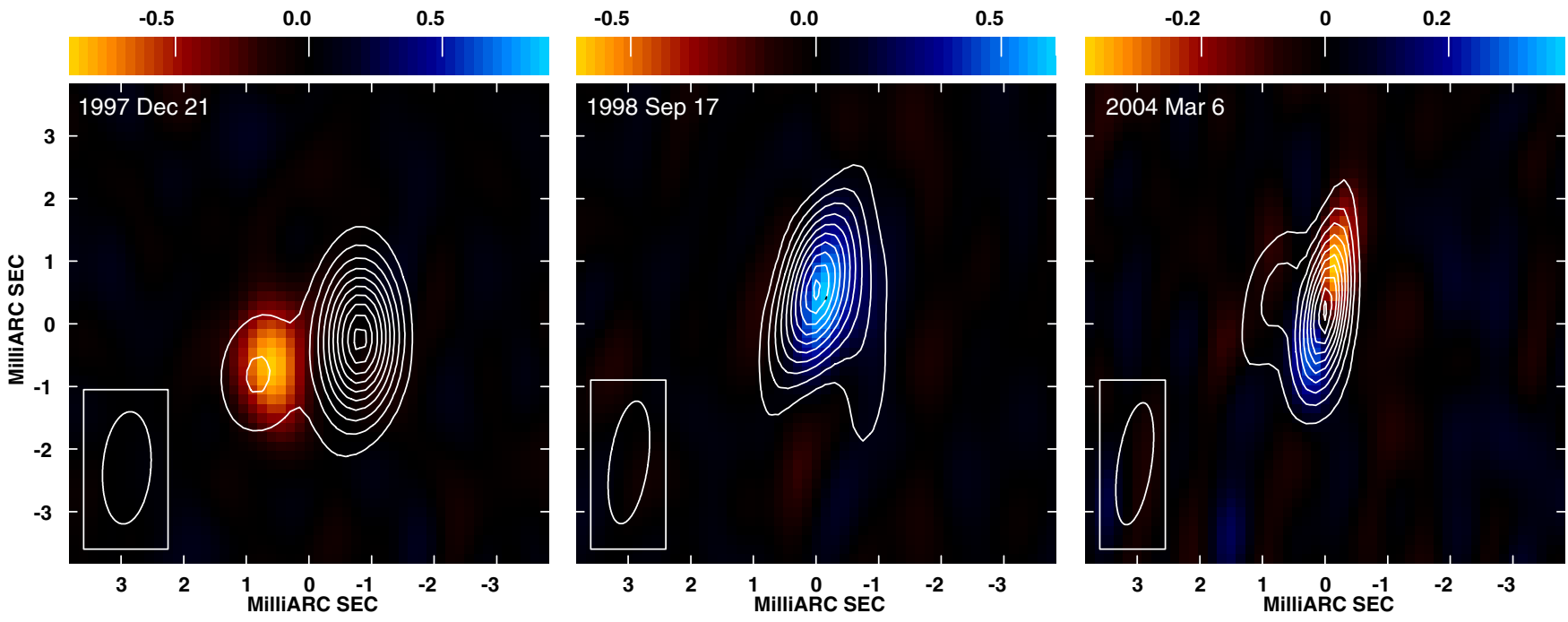

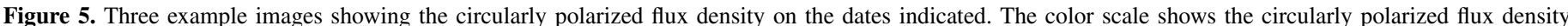

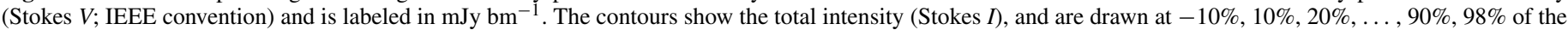

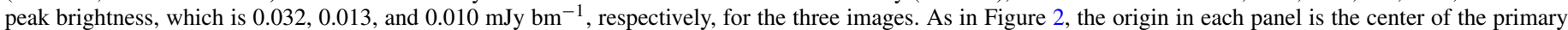
star as determined from the astrometric results in Paper V. North is up and east is to the left.

variety of morphologies, ranging from being largely unresolved to having a clear double and on one occasion (2003 December 6) a possibly triple structure. Although no other RS CVn system has been so extensively observed with VLBI, the images of other systems are generally within the range of the morphologies we observed for IM Peg. The radio emission is highly variable and during most of our VLBI observing sessions the total flux density varied significantly. On occasion, time-resolved images show variations in morphology over several hours. As we have mentioned, apparent motion of IM Peg's emission region, correlated with evolution of its flux density on timescales of $1 \mathrm{hr}$, is definitely seen in our 1997 January 16 session (Lebach et al. 1999), and may be present in at least two of our other observing sessions.

What can be said about the nature of the variability of IM Peg's radio emission? Since IM Peg is a known binary and its orbit is well known from optical spectroscopy (Marsden et al. 2005; Berdyugina et al. 1999b) and from our VLBI astrometry (Papers IV and V), we next investigate whether the radio emission varies significantly with the orbital phase of the binary or in a secular fashion.

\subsection{Does the Radio Brightness Vary with Orbital Phase?}

In Figure 7, we plot flux density against time and also against orbital phase. There is a secular decline in the flux density, with lower flux densities being observed after late 1999. No correlation with orbital phase is apparent. In particular, we find that the times when the flux density is high, presumably because of outbursts, do not occur during any particular part of the orbit. This result is similar to that found for Algol (Mutel et al. 1998), but unlike that found for the RS CVn binary HR 1099, where radio outbursts seem to occur predominantly between the orbital phases of 0.50-0.67 (Slee et al. 2008).

\subsection{Orientation and Elongation of the Radio Emission Region}

As we showed in the previous section, no correlation between the total flux density and the orbital phase is apparent. Since the orbits are nearly circular (Marsden et al. 2005; Berdyugina et al. 1999b; Olah et al. 1998), one might not expect such a correlation

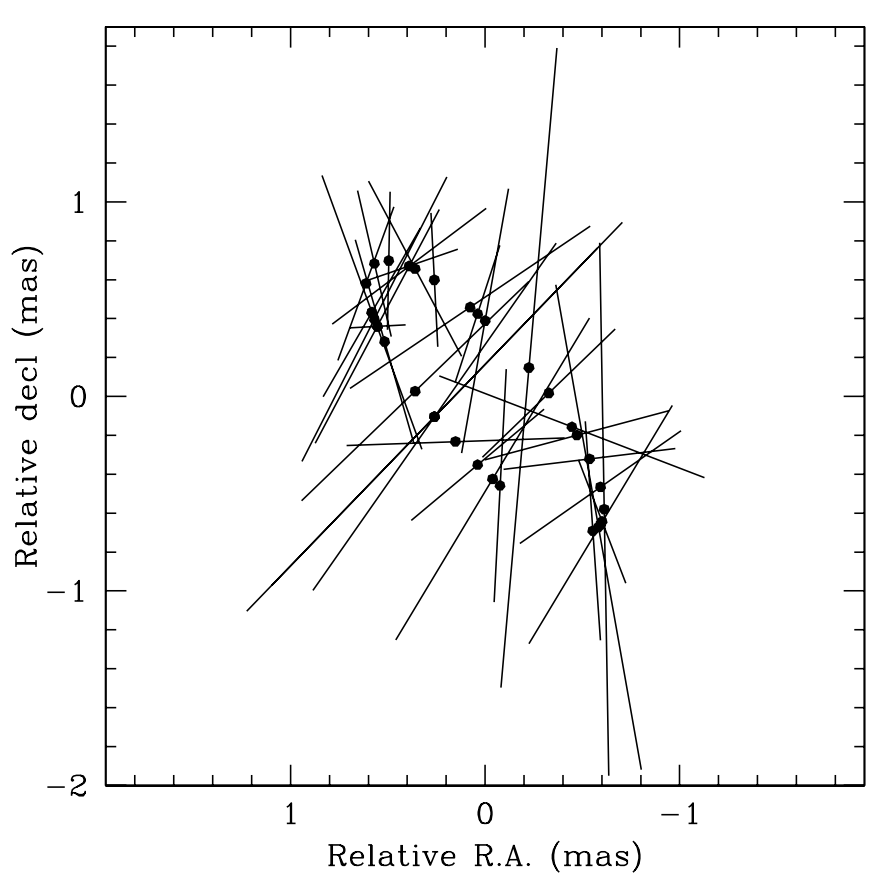

Figure 6. Elongation of the radio emission region of IM Peg as a function of position in the orbit. The dots indicate the projected position in the orbit of the primary star for each of our 35 observing sessions, and the corresponding lines represent the FWHM major axis of an elliptical Gaussian fit to the radio emission of that session. The origin of the coordinate system is the center of the orbit.

if neither eclipsing nor beaming effects are significant. However, the emission region is often extended by as much as the separation between the two stars $(\sim 2$ mas), which leads to the question: is there any correlation between the geometry of the emission region and the orbital phase? For example, a correlation of the amount and/or angle of elongation of the radio emission with orbital phase might be expected if the radio emission originates predominantly along the line between the two stars (as is seen in the Algol system; Peterson et al. 2010; although we note that, unlike IM Peg, Algol is an interacting system for which emission predominately from within the region 

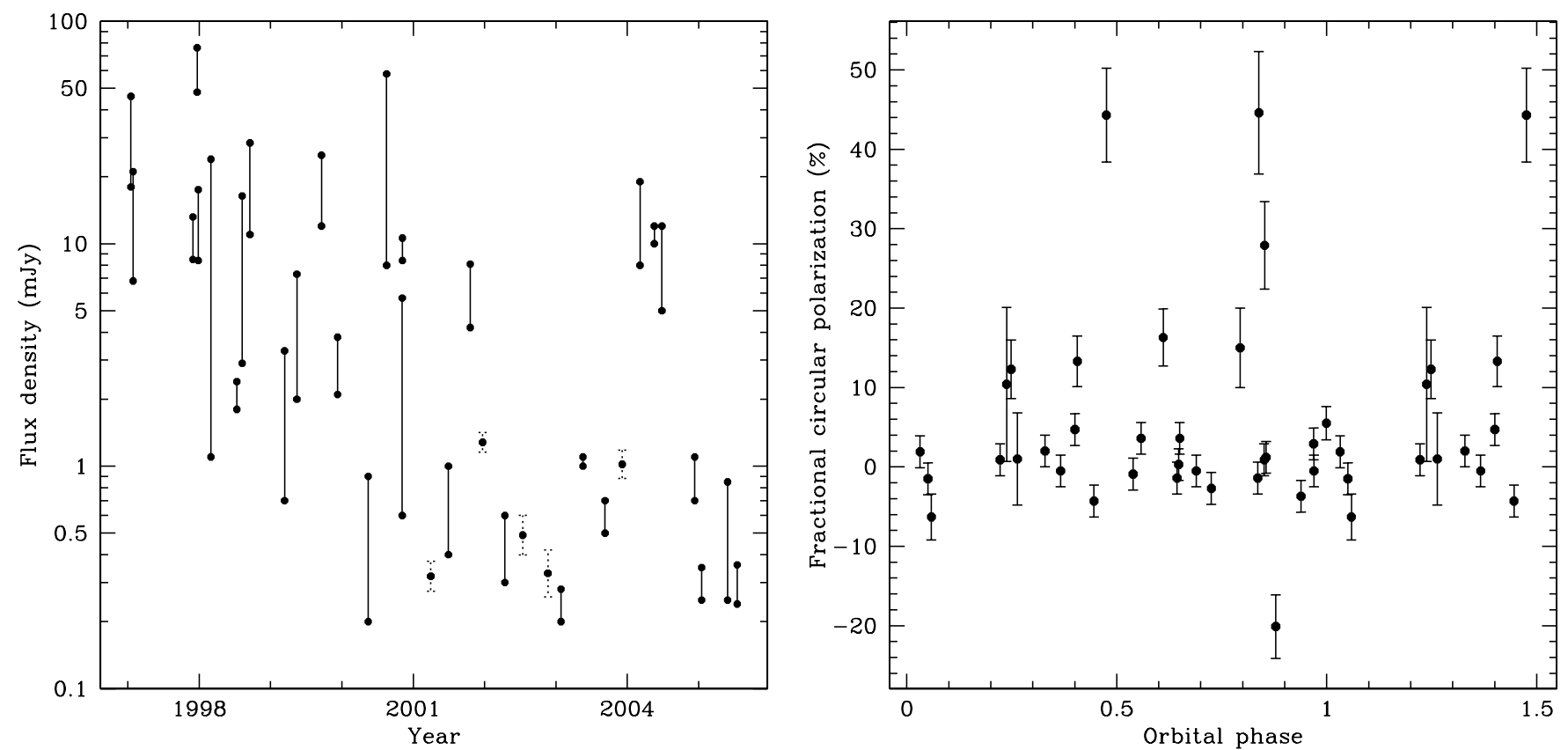

Figure 7. Left panel: the logarithm of the $8.4 \mathrm{GHz}$ total flux density of IM Peg in milli-Janskies, plotted against time. The total flux density often varied within an observing session, and the range of this variation is shown by the vertical lines that connect dots placed at the extreme values of the flux densities during the observing session. In each case where the total flux density did not vary discernibly within the observing interval or where the flux density was determined from VLBI rather than VLA observations, the observational uncertainty $(1 \sigma)$ is shown by a dotted error bar centered on the dot; the fractional observational uncertainties for the other sessions are similar. Right panel: the same flux densities, but plotted against orbital phase (using the orbital parameters of Marsden et al. 2005). Points with orbital phases between 0 and 0.5 are repeated on the right of the plot (with phases between $1.0 \mathrm{ad} 1.5$ ) to make any possible cyclical variation more clearly visible.

between the two stars is physically more plausible). To explore this question, we make use of our Gaussian fits to the emission geometry from Section 3.5 and Table 2, in particular, making use of the p.a., $\theta_{b}$, of the Gaussian fit to the images.

Geometrical and astrophysical considerations suggest that the most likely direction is either along the line from the primary to the secondary or parallel to the orbit normal or perhaps the star's rotation axis if it were different from the orbit normal. In the first case, one would expect that $\theta_{b}$ would vary with the orbital phase, while in the second, one would expect $\theta_{b}$ to remain constant. In Figure 8 , we plot $\theta_{b}$ against time and against orbital phase. Neither hypothesis is well supported. Until approximately $1999.5, \theta_{b}$ is fairly well determined, with the data possibly suggesting a secular variation with $\theta_{b}$ decreasing from $\sim 140^{\circ}$ in 1995.0 to $\sim 100^{\circ}$ by 1999.5 . Thereafter, $\theta_{b}$ is more poorly determined, largely because the total flux densities and hence signal-to-noise ratios were lower due to the aforementioned secular decrease in the average flux density.

In Figure 6, the direction of elongation of the radio emission region does not show a correlation with the line joining the primary to the secondary, implying that the radio emission region does not stretch between the two stars. Since we think it unlikely that the radio emission is associated with the secondary, we do not consider that possibility further. Since we find no evidence that the radio emission is spatially associated with the line joining the two stars, we therefore conclude that the radio emission is associated only with the primary. Indeed, this conclusion is consistent with our astrometry (Paper V and Paper VI), which suggests that the radio emission is associated with the primary. We discuss the possibility that the radio emission is associated with starspots on the primary below in Section 4.5.

As noted in Section 3.5, there is a significant tendency for the emission region to be preferentially extended along
P.A. $=157^{\circ}$, which direction is reasonably close to that of the sky-projected orbital angular momentum, which is at P.A. $130^{\circ} \pm 13^{\circ}$ (Paper V). ${ }^{11}$ The radio emission, therefore, seems to extend preferentially in the projected direction close to that of the orbital angular momentum. This alignment suggests that the emission tends to be associated with the magnetic poles of the star, provided the magnetic poles are near the rotational ones. This location is consistent with the polar-cap model proposed for the Algol system by Mutel et al. (1998).

The average extent of the emission region (as given by the fitted Gaussian FWHM major-axis length, $b$ ) was $1.4 \pm 0.4$ mas, corresponding at $96 \mathrm{pc}$ to $(1.9 \pm 0.6) \times 10^{12} \mathrm{~cm}$, or $1.1 \pm$ 0.3 times the star's diameter (for which we again use the value of $\sim 27 R_{\odot}$ from Berdyugina et al. 1999b). This result suggests that the bulk of the radio emission generally occurs within an area not much larger in size than the stellar disk.

\subsection{Rapid Evolution of the Radio Structure}

By dividing an observing session temporally, we can assess any possible rapid evolution of the image geometry. A rapid change in the apparent position accompanied by a large change in flux density is seen in several sessions. The most dramatic example was the already mentioned change in apparent position of $\sim 0.9$ mas over a period of $1.4 \mathrm{hr}$ on 1997 January 16 (see Lebach et al. 1999). Such a change in apparent position could be due either to (1) fast motion at $\sim 1000 \mathrm{~km} \mathrm{~s}^{-1}$ of flare-energized

\footnotetext{
11 As we mention in Section 3.5 above, the systematic uncertainty on the average angle of elongation of the radio emission likely dominates the statistical one of $\pm 7^{\circ}$. We can therefore not make a more definitive statement as to whether the average direction of elongation of the radio emission lies along the orbit normal within the uncertainties or not. We note that any bias in our estimate of the elongation of the emission region toward the average elongation of the restoring beam at P.A. $=174^{\circ}$ would bring it closer to the angle of the orbit normal of $130^{\circ} \pm 13^{\circ}$.
} 

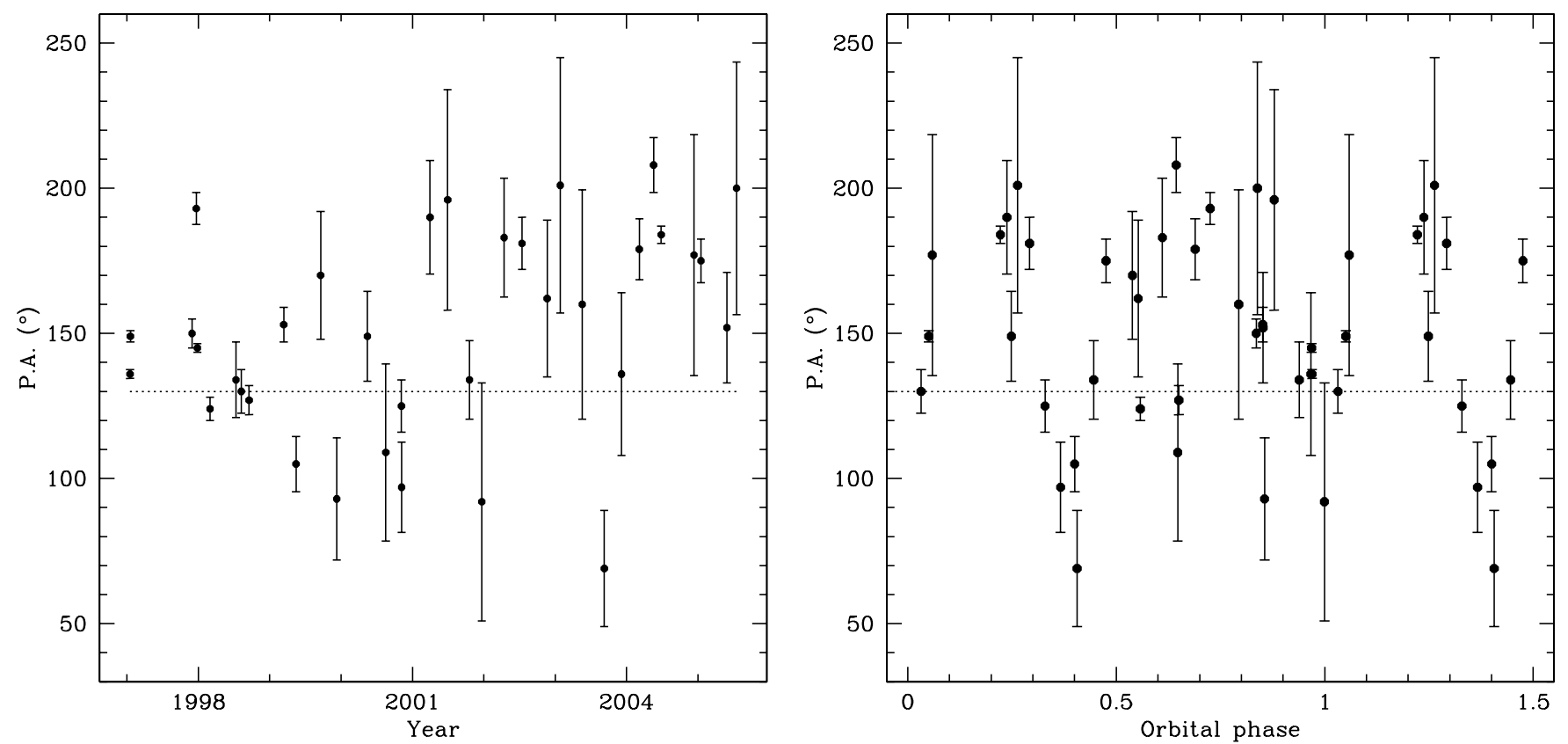

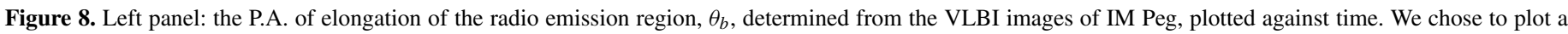

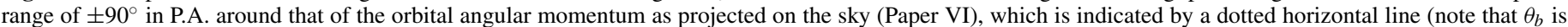

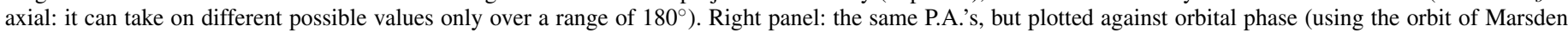

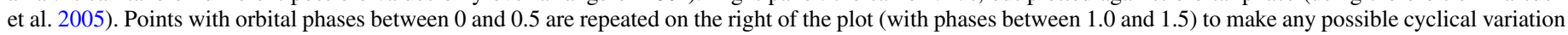
more clearly visible.

electrons in a single magnetic-loop structure or (2) two spatially distinct components separated by $\sim 1$ mas, with a rapid change in their relative brightness. Given the elongation of the emission region for the 1997 Jan observing session (see Figure 2), the latter is more plausible. However, for the 1998 March and 1998 August sessions, in which the emission region is quite compact, motion of the energized electrons is a likely possibility.

We see double (and in one case, an apparent triple) structure in $\sim 25 \%$ of our images. Images made from temporal subsets of some of our observing sessions, for example those shown in Figure 3, show that the double structure usually persists over periods $>6 \mathrm{hr}$. We found in Section 4.2 above that, on average, the extent of the radio emission on the sky was comparable to the size of the stellar disk. On occasion, though, the extent of the radio emission (as given by the FWHM of an elliptical Gaussian fitted to the image) is up to about twice the stellar diameter. ${ }^{12}$ It seems plausible therefore that most of the radio emission occurs not far from the stellar surface, but on occasion, relatively bright radio emission is generated at distances of up a stellar diameter above the surface.

It is likely that the radio emission originates in active regions, e.g., magnetic-loop structures, approximately a stellar radius in size (see, e.g., Peterson et al. 2010; Mullan et al. 2006; Franciosini et al. 1999; Lestrade et al. 1988), which are rooted on the surface of the star. Indeed, IM Peg is of the spectral type $\mathrm{K} 2$, and Mullan et al. (2006) show that the largest coronal loops, with sizes up to two stellar radii, occur in stars of type K2 or later. Although the radio brightness of each emission region is often variable on timescales of $\sim 1 \mathrm{hr}$ or less, the regions themselves appear longer lived, since the radio emission does not generally

\footnotetext{
12 We note that the largest value for an FWHM major axis of the radio emission was observed on 2005 January 15 and was 3.3 mas, corresponding to 2.5 stellar diameters. That particular value, however, is rather uncertain as IM Peg was very weak for that session. Of the better-determined major-axis values, the largest one corresponds to $\sim 2.2$ times the diameter of the stellar disk.
}

seem to move on scales of the stellar radius over periods of a few hours. The rotation and orbital motion of the primary will produce proper motions of $\sim 10 \mu \mathrm{as} \mathrm{hr}^{-1}$, which is below what can be reliably determined from our images. The lack of motions on the short timescales of the flux-density variations suggests that more rapid bulk or pattern motion, for example due to plasma moving along the magnetic-loop structures, are not prevalent or at least generally occur on scales smaller than the stellar radius.

\subsection{Fourier Analysis of Quantities Derived from Images}

The near-equality between the photometric period of IM Peg (variable between 23.8 and 25.2 days; see Strassmeier et al. 1997) with the spectroscopic one of 24.64877 days suggests that the rotation of IM Peg primary is tidally locked to its orbital motion (note that, given the variable nature of the dark spots on the surface of the star, an exact equivalence between the photometric and spectroscopic periods is not expected). Both the lack of any clear correlations between either the location of the emission relative to the center of the star, or the direction of elongation of the emission region with the orbital phase, and the observations of apparent motion on hour timescales therefore suggest that the brightest emission does not always emanate from a region centered on a single spot on the rotating surface of the star.

Optical Doppler imaging of IM Peg's surface has shown that there are dark spots, which cover $\gtrsim 15 \%$ of the star's surface (Berdyugina \& Marsden 2006; Berdyugina et al. 2000). These dark spots are presumably the cause of the photometric variability. The photometric period, however, does not correspond precisely to the orbital one. The photometric period is variable, ranging between $\sim 24$ and $\sim 25$ days between 1993 and 1999 (Strassmeier et al. 1997; Berdyugina et al. 2000). In fact, multiband photometry can be used to constrain the spot population independent of Doppler imaging and gives generally consistent 


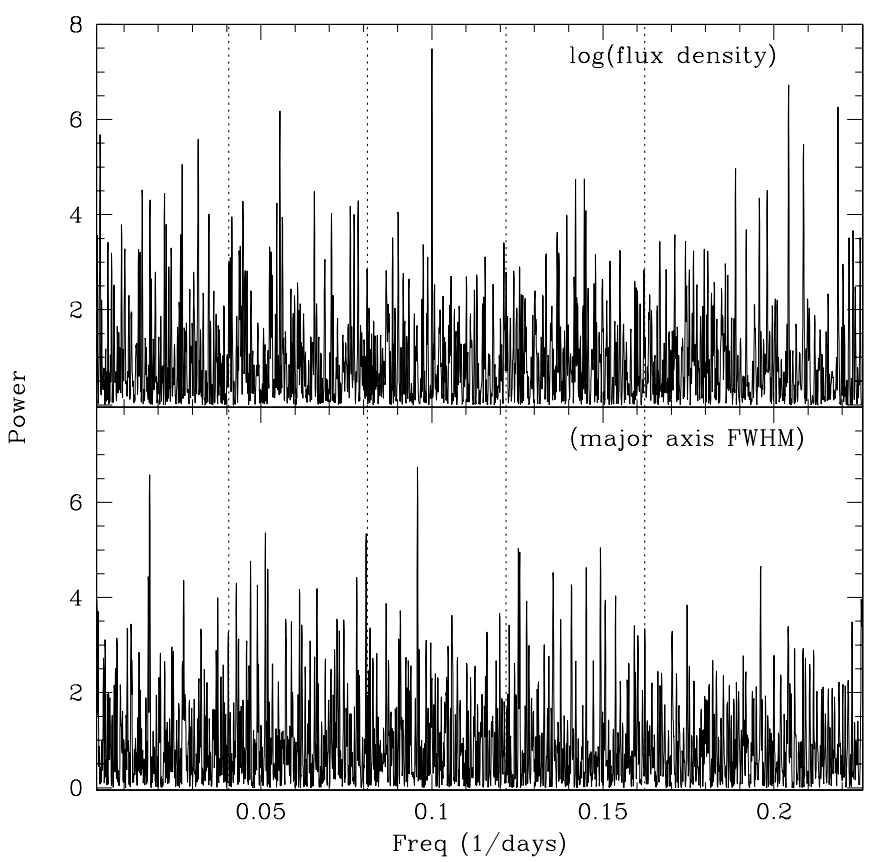

Figure 9. Upper panel: normalized Lomb-Scargle periodogram of the $\log$ of the total flux density of IM Peg derived from our 35 observing sessions of observations. The dotted lines indicate the spectroscopic binary frequency of $(1 / 24.64877)$ day $^{-1}$ and its first three harmonics. Lower panel: a normalized Lomb-Scargle periodogram of the maximum angular extent of the radio emission, as parameterized by the FWHM major axis of an elliptical Gaussian fitted to the radio images.

results (Zellem et al. 2010). Over the period 1996-1999, the dark spots responsible for the photometric variation occur preferentially at two active longitudes ${ }^{13}$ on opposite hemispheres (in longitude), but seem to drift in longitude by about 0.05 day $^{-1}$, resulting in a photometric period which can vary slightly from the spectroscopic one.

Do our measurements show evidence of periodicities other than the orbital one? To identify any possible periodicities in the radio data, we produced normalized Lomb-Scargle periodograms (Scargle 1982) of several quantities derived from the radio observations, namely, the total flux density, the fractional variation of the flux density within the observing session, the cosine and sine of the P.A. of elongation, the major axis length of an elliptical Gaussian fit to the radio images, and the average (over an observing session) degree of circular polarization. In none of these periodograms did we find any significant periodicity. All the peaks seen have chance probabilities of $30 \%$ or higher (probabilities are calculated according to Scargle 1982). As an example, we show in Figure 9 the periodograms for the total flux density and the maximum angular extent of the radio emission as parameterized by the FWHM major axis of an elliptical Gaussian fit to each radio image. No peaks higher than would be expected by chance are observed, and the highest peaks observed in either periodogram are not near the orbital frequency or its first three harmonics. No significant peaks at longer periods, such as the 294 day (frequency $=\sim 0.0035$ day $^{-1}$ ) Rieger-like flaring periodicity found in the RS CVn system UX ARI (Massi 2007; Massi et al. 2005), are observed. We note, however, that there are local maxima near some of the harmonics of the orbital frequency, indicating that there may well be some periodic behavior, but

\footnotetext{
13 The stellar longitude is defined by assuming tidally-locked rotation, with
} longitude zero being for the meridian facing the secondary. not at a level reliably distinguishable with our data from mere random variation. In particular, there is moderately high peak near the second harmonic in the periodogram for the majoraxis length, at a frequency of 0.0808 day $^{-1}$ or $1.99 \times$ the orbital one of $0.040570 \mathrm{day}^{-1}$. This peak is the fourth highest in the periodogram. If the location of the periodogram peaks were random, the chance that one of the four highest peaks would fall within $1 \%$ of the first four harmonics of the orbital frequency would be $\sim 3 \%$. If the periodicity is associated with the projection onto the sky of some dimension which rotates with the stars in their orbit, then geometrical considerations in fact suggest a frequency double the orbital one, as is observed. We also note that the photometric period seems to be slightly different than the orbital one. Berdyugina et al. (2000) found a spot rotation frequency of 0.997 times the orbital one during the period 1996-1999; its second harmonic would be very close to the periodogram peak in question.

In conclusion, no statistically significant periodicities are visible in the characteristics of the radio emission: its total flux density, its short timescale variability, or its spatial extent. No significant periodicities are present in the astrometric residuals either (see Paper V). We note, however, that there is evidence, albeit inconclusive, for some geometrical effect on the extent of the radio emission region associated with either the rotation of the binary or more likely the rotation of the starspots on the primary star.

\subsection{Comparison of Radio Images with Optical Doppler Imaging}

Optical spectra from short exposures of $\lesssim 20$ minutes allow the imaging of portions of the star's photosphere using Doppler imaging (e.g., Berdyugina \& Marsden 2006; Berdyugina et al. 2000, 1999b). As mentioned earlier, such imaging shows that the dark spots occur primarily at one or more long-lived active longitudes on the surface of the star. Ribárik et al. (2003) came to a similar conclusion by analyzing the photometric data. The optical observations showed that the most intense spots usually occur on the side toward the secondary, i.e., at a stellar longitude near 0 , although, as noted above, the active longitudes were found to change slowly with time (Marsden et al. 2007; Berdyugina \& Marsden 2006; Ribárik et al. 2003; Berdyugina et al. 2000), likely as a result of differential rotation of the stellar surface, as we see in the Sun, and as has been observed in a different RS CVn star, II Pegasi (Roettenbacher et al. 2011). The dark spots also seem more likely to occur at stellar latitudes nearer the pole than the equator (Berdyugina \& Marsden 2006; Berdyugina et al. 2000).

As our astrometric fit gives a good estimate of the expected position of the center of the star for each observing session, can we calculate the location of the brightness peak of the radio emission as projected onto the star's surface for comparison with the location of the optical dark spots? Given that the height of the radio-emission peak above the stellar surface is unknown, the stellar longitude and latitude cannot be directly obtained. However, as we showed in Paper VI, the scatter in the positions of the peak brightness points relative to the estimated center position of the star was consistent with a distribution having a scale height of only $\sim 0.2$ stellar radii above the surface of the star. We also concluded there that "spillover" emission, that is where the emission originates on the side of the star away from us, but enough of it spreads over the limb to be detected, was responsible for only a relatively small fraction of the observed radio brightness peaks. 

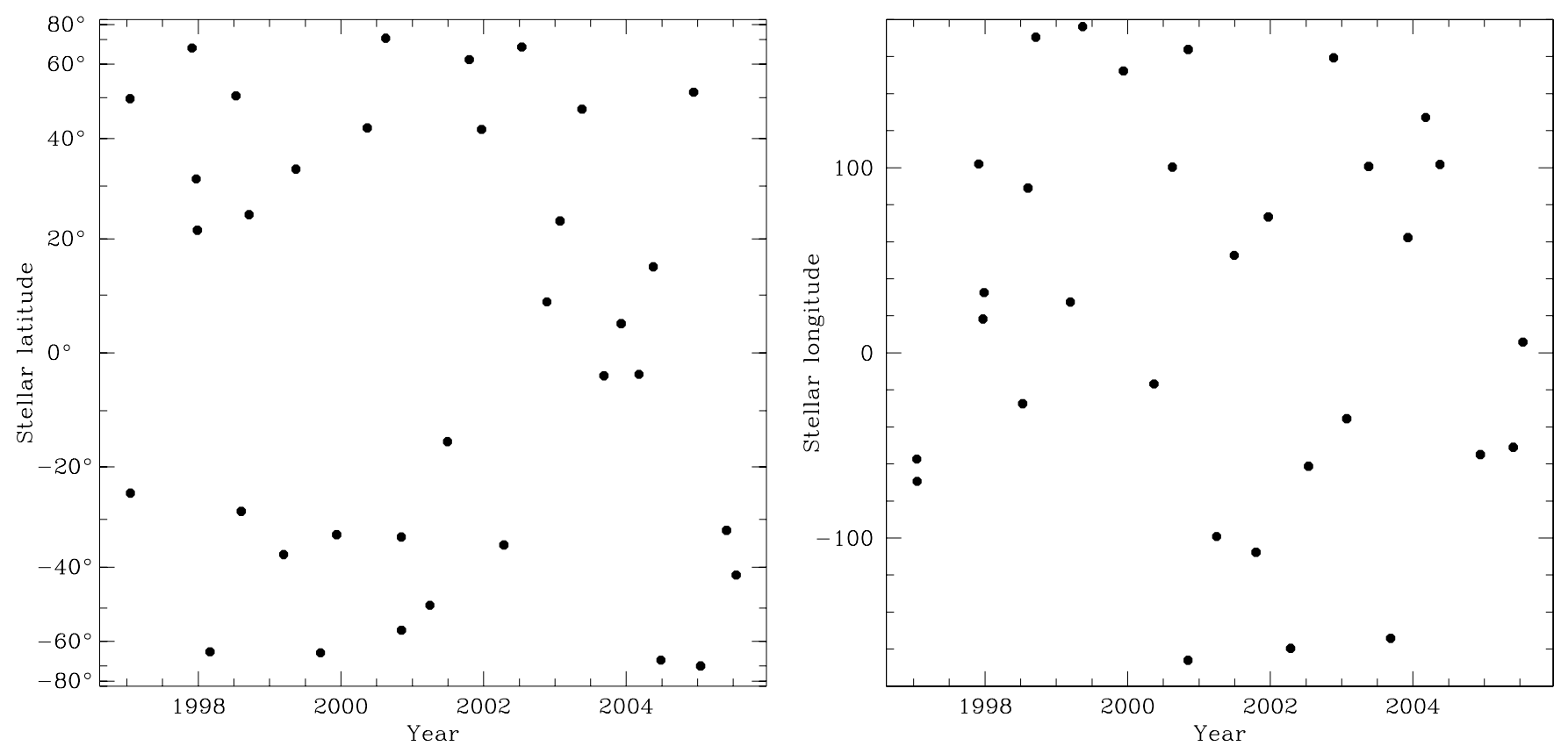

Figure 10. Latitude and longitude on the surface of the star corresponding to the peak brightness point of the radio emission. We calculate the latitude and longitude by assuming the star's center to be at the position given by our astrometric fit, and by taking the star's angular radius to be 0.64 mas (see Section 4.5 for the assumptions used in calculating the stellar latitude and longitude). Since the longitude becomes degenerate near the pole of the star, we omit in the right panel the four points which were $<0.1$ mas from the pole on the sky.

We therefore make the simplifying approximations that the emission peak is located exactly on the stellar surface and is confined to the half of the surface facing us. With these approximations, we can then calculate stellar latitude and longitude of the emission peak for each observing session except for those where the brightness peak falls outside the stellar disk. For those sessions, we calculate the latitude and longitude for the nearest point on the limb of the star. We again use the value of $13.3 R_{\odot}$ (Berdyugina et al. 1999b), which corresponds to 0.64 mas, for the radius of the star. Although the calculation of stellar latitude and longitude of the brightness peak is approximate and could be seriously in error for a few sessions because of the above simplifying assumptions, in general our estimates should be adequate for our purpose of determining whether the emission regions are preferentially associated with some parts of the star's surface. We plot our values of the stellar latitude and longitude of the brightness peak against time in Figure 10.

The radio emission shows no strong preference for any particular stellar longitudes. In particular, we find no significant preponderance of radio emission near zero longitudes (see Figure 10). Eliminating indeterminate longitudes associated with high latitudes, we are left with 31 values for the longitude of the brightness peak. Of these, 17 , or $55 \% \pm 12 \%$, have longitudes in the range $-90^{\circ}$ to $90^{\circ}$ (where longitude zero faces the secondary), which is consistent with the spot longitude being random.

As we already found in Paper VI, the radio brightness peak may show a preference for stellar latitudes nearer the poles. For example, $8 \pm 3$ points have a latitude either $\geqslant \pm 60^{\circ}$ or $\leqslant \pm-60^{\circ}$, whereas only 5 would be expected by chance.

The optical dark spots also show a preference for latitudes near the poles (Marsden et al. 2007; Berdyugina \& Marsden 2006), suggesting that there may be a connection between the regions of bright radio emission and the optical dark spots. Although the localization on the star's surface of the radio bright regions and the optical dark spots is not of sufficient accuracy to warrant definitive conclusions, the evidence suggests that the radio-bright regions do not directly correspond to the optical dark spots, since the radio-bright regions do not seem to show the same preferred longitudes as do the dark spots. Nonetheless, the dark spots may represent the locations from which at least some short-lived radio-bright flares emanate, as on the Sun.

\subsection{The Nature of the Radio Emission}

Our radio data suggest that the radio emission is highly variable temporally and spatially, and is at least on occasion, localized in regions covering only a fraction of the stellar surface. This picture is supported by the observation of time variability on scales much shorter than the orbital period, and of rapid ( $\lesssim 1 \mathrm{hr}$ ) positional variability accompanied by rapid changes in flux density. In other words the radio emission seems likely related to some rapid energy release localized on the stellar surface.

Indeed, the total radio emission is likely due to several distinct but temporally overlapping energy releases, or flares, consistent with the model proposed for RS CVn radio emission by Franciosini \& Chiuderi-Drago (1994) and Mutel et al. (1985). The flares have timescales of only a few hours, with individual flares originating at different locations on, or above, the surface of the star. On occasion, more than one such spot is present and we observe double, or possibly triple, structure. The radio emission is extended preferentially in a polar direction, perhaps because of magnetic field lines extending also in that direction. On average the angular extent of the emission region is similar to that of the stellar disk, although it can be larger. This observation suggests that the radio emission mostly occurs near the stellar surface, but on occasion also occurs significantly above it.

Although the short timescales and the prevalence of multiple structure suggest that the radio emission seems concentrated in small regions of the star's surface, we have no evidence that 
the radio flare spots are associated with the optical dark spots on the stellar surface. It may, however, arise from extended magnetic-loop structures whose feet are fixed to these active surface regions. The occasional release of magnetic energy stored at or below the surface in the long-lived spot regions could energize electrons trapped in the loops, which then radiate via the gyrosynchrotron mechanism.

The average flux density of IM Peg decreased during our 8.5 year observing period. During the first few years of our observing program, the above-mentioned flares were more frequent, occurring sometimes within just a few hours of one another (see Figure 1). Toward the later part of our program, flares were much less frequent, separated perhaps by several days. If there is a steady-state population of radio-emitting electrons, our observations set a limit on this quiescent emission at a level $\lesssim 0.3 \mathrm{mJy}$.

\section{SUMMARY}

We discuss the unprecedented series of 35 VLBI radio images of the RS CVn star IM Peg at $8.4 \mathrm{GHz}$, taken over a period of 8.5 years. These astrometric and imaging VLBI observations were undertaken because IM Peg was chosen as the guide star for $G P-B$. As well as the imaging VLBI observations, we obtained total flux-density measurements using the VLA. Our astrometric solution for the star's proper motion, orbit, and parallax allows us to align the images in a star-centered frame. We find the following.

1. The radio emission from the star is rapidly variable. During most of our observing sessions, the total flux density varied by a factor of at least two over the course of several hours.

2. Significant circular polarization was observed for about half of our observing sessions. The fractional circular polarization was also rapidly variable and had a tendency to be higher when the total flux density was lower. For epochs with high circular polarization and low flux density, the circular polarization was predominately positive (IEEE convention).

3. The morphology of the radio emission is also variable. Mostly the emission region has a single-peaked structure, but about $25 \%$ of the time, we observed two (and on one occasion three) peaks. On average, the emission region is elongated by $\sim 1.4 \pm 0.4$ mas (FWHM).

4. On average, the emission region is comparable in size to the disk of the primary star. It is also, on average, somewhat more extended in the direction of the sky-projected angular momentum of the binary orbit. The radio emission is likely associated with one or more regions on or above the surface of the star.

5. We searched for, but did not find, any sign of periodic behavior, either at the known orbital period (24.64877 days) or at any other periods. In particular, neither flux density, degree of circular polarization, degree, or angle of elongation of the emission region showed any significant periodicities.

6. The regions of brightest radio emission show no preference for any particular stellar longitudes, unlike the dark spots seen in the optical. Nonetheless, like the optical dark spots, the regions of bright radio emission regions show a preference for stellar latitudes near the poles. Despite a likely relationship between the mechanisms, the regions of brightest radio emission are short-lived, and do not seem to be directly associated with the long-lived optical dark spots.
7. The radio emission may arise due to short-lived flares, with each flare occupying only a small part of the stellar surface. These flares may be associated with a magnetic field which has its strongest component along the direction of the rotational axis.

This research was primarily supported by NASA, through a contract with Stanford University to SAO and a subcontract from SAO to York University. The National Radio Astronomy Observatory is a facility of the National Science Foundation operated under cooperative agreement by Associated Universities, Inc. The DSN is operated by JPL/Caltech, under contract with NASA. This research has made use of the United States Naval Observatory (USNO) Radio Reference Frame Image Database (RRFID). We have also made use of NASA's Astrophysics Data System Abstract Service, developed and maintained at SAO. Jeff Cadieux and Julie Tome helped with the data reduction during their tenure as students at York University. Finally, we thank the anonymous referee for useful comments.

\section{REFERENCES}

Baars, J. W. M., Genzel, R., Pauliny-Toth, I. I. K., \& Witzel, A. 1977, A\&A, 61,99

Bartel, N., Bietenholz, M. F., Lebach, D. E., et al. 2012, ApJS, 201, 3 (Paper III) Bartel, N., Ransom, R. R., Bietenholz, M. F., et al. 2008, in IAU Symp. Vol. 248, A Giant Step: From Milli- to Micro-arcsecond Astrometry, ed. W. J. Jin, I. Platais, \& M. A. C. Perryman (Cambridge: Cambridge Univ. Press), 190

Batschelet, E. 1981, Circular Statistics in Biology (London: Academic)

Beasley, A. J., \& Güdel, M. 2000, ApJ, 529, 961

Berdyugina, S. V., Berdyugin, A. V., Ilyin, I., \& Tuominen, I. 2000, A\&A, 360, 272

Berdyugina, S. V., Ilyin, I., \& Tuominen, I. 1999a, A\&A, 349, 863

Berdyugina, S. V., Ilyin, I., \& Tuominen, I. 1999b, A\&A, 347, 932

Berdyugina, S. V., \& Marsden, S. C. 2006, in ASP Conf. Ser. 358, Solar Polarization 4, ed. R. Casini \& B. W. Lites (San Francisco, CA: ASP), 385

Bietenholz, M. F., Bartel, N., \& Rupen, M. P. 2003, ApJ, 597, 374

Briggs, D. S. 1995, BAAS, 27, 1444

Briggs, D. S., Schwab, F. R., \& Sramek, R. A. 1999, in ASP Conf. Ser. 180, Synthesis Imaging in Radio Astronomy II, ed. G. B. Taylor, C. L. Carilli, \& R. A. Perley (San Francisco, CA: ASP), 127

Drake, S. A., Simon, T., \& Linsky, J. L. 1989, ApJS, 71, 905

Dulk, G. A. 1985, ARA\&A, 23, 169

ESA 1997, The Hipparcos and Tycho Catalogues (ESA SP-1200; Noordwijk: ESA)

Franciosini, E., \& Chiuderi-Drago, F. 1994, Radiophys. Quantum Electron., 37, 403

Franciosini, E., Massi, M., Paredes, J. M., \& Estalella, R. 1999, A\&A, 341, 595

Hall, D. S. 1976, in ASSL Vol. 60: IAU Colloq. 29: Multiple Periodic Variable Stars, ed. W. S. Fitch (Dordrecht: Reidel), 287

Hjellming, R. M. 1988, in Galactic and Extragalactic Radio Astronomy, ed. G. L. Kellermann \& K. I., Verschuur (Berlin: Springer), 381

Jones, K. L., Brown, A., Stewart, R. T., \& Slee, O. B. 1996, MNRAS, 283, 1331

Lebach, D. E., Bartel, N., Bietenholz, M. F., et al. 2012, ApJS, 201, 4 (Paper IV)

Lebach, D. E., Ratner, M. I., Shapiro, I. I., et al. 1999, ApJ, 517, L43

Lestrade, J.-F., Jones, D. L., Preston, R. A., et al. 1995, A\&A, 304, 182

Lestrade, J.-F., Mutel, R. L., Preston, R. A., \& Phillips, R. B. 1988, ApJ, 328, 232

Marsden, S. C., Berdyugina, S. V., Donati, J.-F., Eaton, J. A., \& Williamson, M. H. 2007, Astron. Nachr., 328, 1047

Marsden, S. C., Berdyugina, S. V., Donati, J.-F., et al. 2005, ApJ, 634, L173

Massi, M. 2007, Mem. Soc. Astron. Ital., 78, 247

Massi, M., \& Aaron, S. 1999, A\&AS, 136, 211

Massi, M., Felli, M., Pallavicini, R., et al. 1988, A\&A, 197, 200

Massi, M., Neidhöfer, J., Carpentier, Y., \& Ros, E. 2005, A\&A, 435, L1

Mullan, D. J., Mathioudakis, M., Bloomfield, D. S., \& Christian, D. J. 2006, ApJS, 164, 173

Mutel, R. L., Lestrade, J. F., Preston, R. A., \& Phillips, R. B. 1985, ApJ, 289, 262

Mutel, R. L., Molnar, L. A., Waltman, E. B., \& Ghigo, F. D. 1998, ApJ, 507, 371 
Olah, K., Marik, D., Houdebine, E. R., Dempsey, R. C., \& Budding, E. 1998, A\&A, 330, 559

Paredes, J. M. 2005, in EAS Publications Series, Radio Astronomy from Karl Jansky to Microjansky, ed. L. I. Gurvits, S. Frey, \& S. Rawlings (Les Ulis: EDP Sciences), 187

Peterson, W. M., Mutel, R. L., Güdel, M., \& Goss, W. M. 2010, Nature, 463, 207

Peterson, W. M., Mutel, R. L., Lestrade, J.-F., Güdel, M., \& Goss, W. M. 2011, ApJ, 737, 104

Ransom, R. R., Bartel, N., Bietenholz, M. F., et al. 2002, ApJ, 572, 487

Ransom, R. R., Bartel, N., Bietenholz, M. F., et al. 2003, ApJ, 587, 390

Ransom, R. R., Bartel, N., Bietenholz, M. F., et al. 2005, in ASP Conf. Ser. 340, Future Directions in High Resolution Astronomy, ed. J. Romney \& M. Reid (San Francisco, CA: ASP), 506
Ransom, R. R., Bartel, N., Bietenholz, M. F., et al. 2012a, ApJS, 201, 2 (Paper II) Ransom, R. R., Bartel, N., Bietenholz, M. F., et al. 2012b, ApJS, 201, 6 (Paper VI)

Ratner, M. I., Bartel, N., Bietenholz, M. F., et al. 2012, ApJS, 201, 5 (Paper V)

Rayner, D. P., Norris, R. P., \& Sault, R. J. 2000, MNRAS, 319, 484

Ribárik, G., Oláh, K., \& Strassmeier, K. G. 2003, Astron. Nachr., 324, 202

Roettenbacher, R. M., Harmon, R. O., Vutisalchavakul, N., \& Henry, G. W. 2011, AJ, 141, 138

Scargle, J. D. 1982, ApJ, 263, 835

Shapiro, I. I., Bartel, N., Bietenholz, M. F., et al. 2012, ApJS, 201, 1 (Paper I)

Slee, O. B., Wilson, W., \& Ramsay, G. 2008, PASA, 25, 94

Strassmeier, K. G., Bartus, J., Cutispoto, G., \& Rodono, M. 1997, A\&AS, 125, 11

Zellem, R., Guinan, E. F., Messina, S., et al. 2010, PASP, 122, 670 